LBNL-54447

\title{
Energy Manager Design for Microgrids
}

\author{
Prepared for the \\ California Energy Commission \\ Principal Authors \\ Ryan Firestone and Chris Marnay \\ Ernest Orlando Lawrence Berkeley National Laboratory \\ 1 Cyclotron Road, MS 90R4000 \\ Berkeley CA 94720-8136
}

January 2005

The work described in this paper was coordinated by the Consortium for Electric Reliability Technology Solutions and funded by the California Energy Commission, Public Interest Energy Research Program, under Work for Others Contract No. 150-99003, Am\#1 and by the U.S. Department of Energy under Contract DE-AC03-76SF00098. 



\section{Preface}

The technical and financial feasibility and desirability of microgrids has been shown in simulation and on paper. In order to demonstrate them in practice, an energy manager (EM) for control of microgrid equipment is needed. An all-knowing EM is not possible, an extremely information rich and intelligent but expensive EM might not be the optimal choice, and yet an EM that is too simple threatens several perceived benefits of DER. Both art and science will be required to develop the optimal EM and microgrid for a given site. This report serves to introduce the science. Further work should serve to develop this science and to gain experience on actual systems from which the art will emerge. 



\section{Table of Contents}

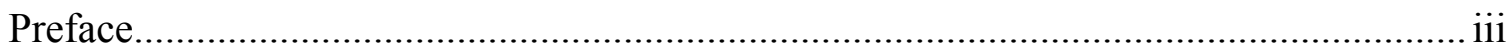

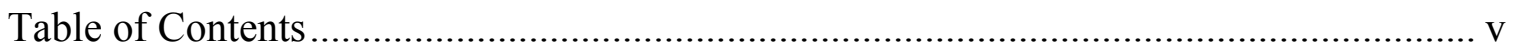

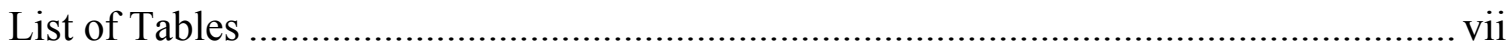

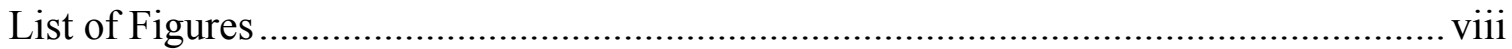

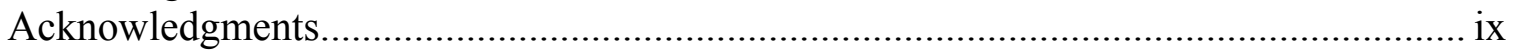

List of Acronyms, Abbreviations, and Definitions ........................................................ xi

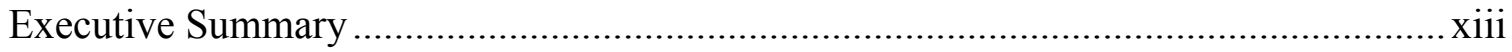

1. Introduction............................................................................................... 1

2. The CERTS Microgrid Concept and the Need For an EM System ............................ 5

2.1 Controls For the CERTS Microgrid............................................................. 5

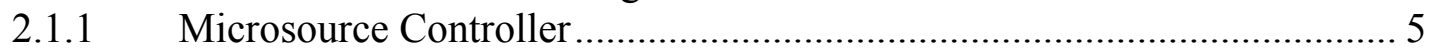

2.1.2 Protection Coordinator..................................................................... 5

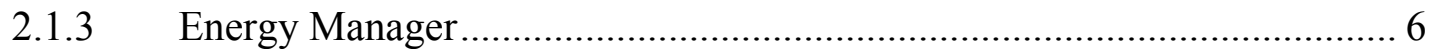

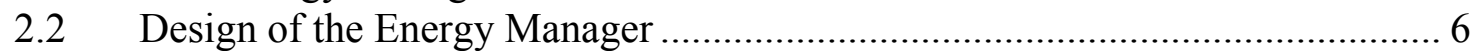

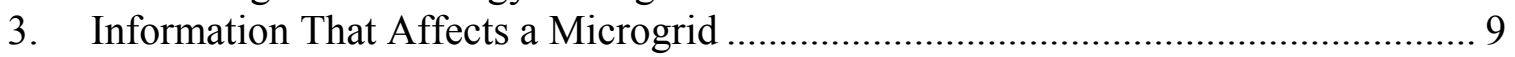

$3.1 \quad$ Tariff and Regulatory Information............................................................. 9

3.2 Equipment Performance and Monitoring........................................................ 10

3.3 Atmospheric Predictions and Data................................................................ 10

3.4 Load Prediction and System Models ………................................................ 10

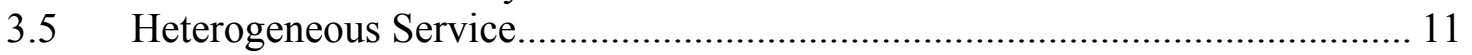

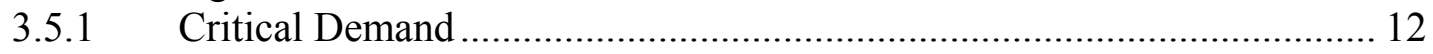

3.5.2 Curtailable Demand ...................................................................... 12

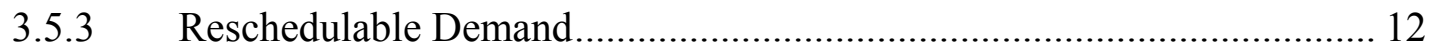

4. Control Strategies Available for Microgrids..................................................... 15

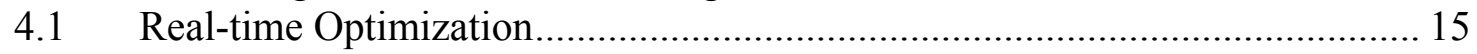

4.2 Expert Systems and Fuzzy Expert Systems ...................................................... 16

4.3 Decentralized and Hierarchical Control........................................................ 17

5. The Dynamically Adaptable Microgrid .............................................................. 19

5.1 Requirements on DER Equipment for a Dynamically Adaptable Microgrid ... 19

5.2 Requirements on the EM for a Dynamically Adaptable Microgrid.................. 20

5.2.1 Supply 0Side: DER Equipment ...................................................... 20

5.2.2 Demand Side................................................................................. 20

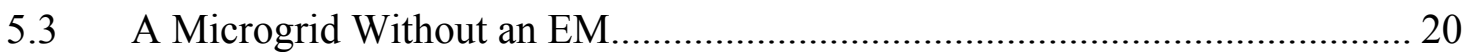

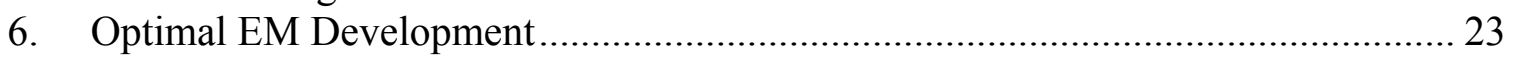

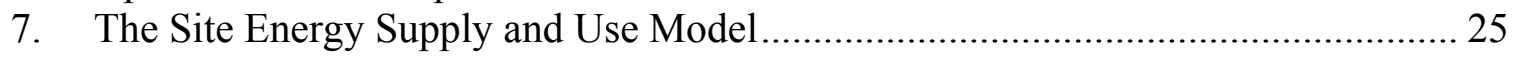

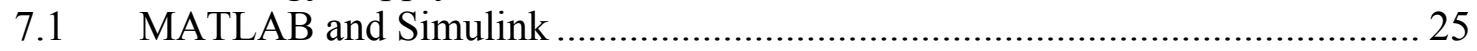

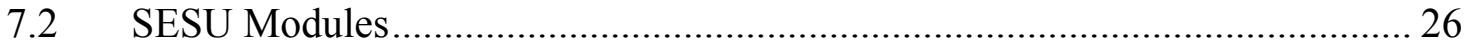

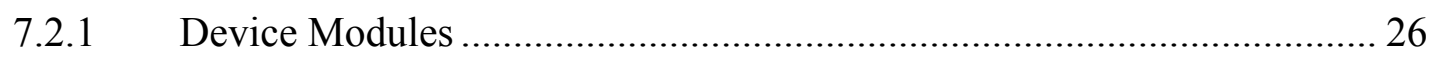

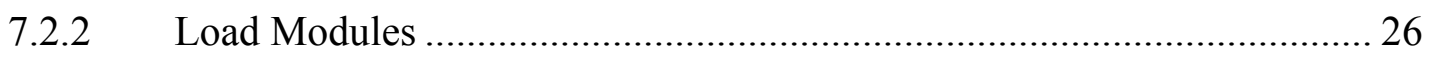

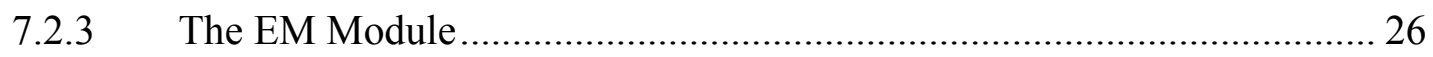

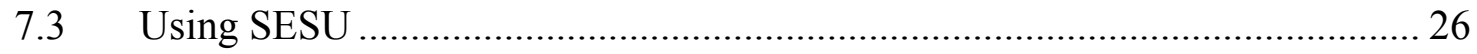

7.4 Related Work in the Modular Modeling of Configurable Systems .................. 27

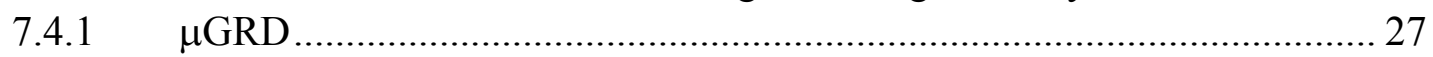

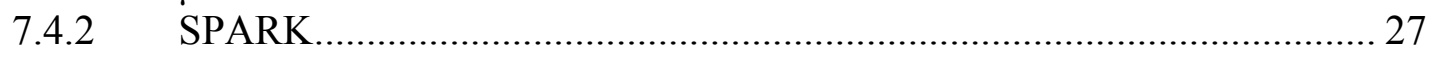




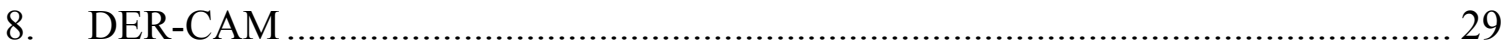

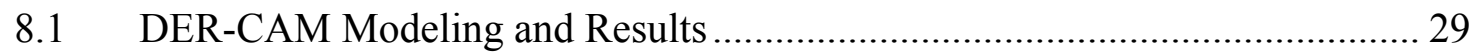

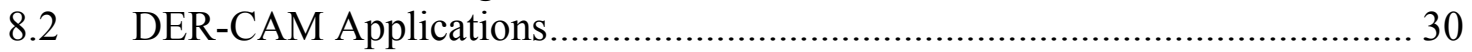

8.3 The Need for More Detailed Modeling ............................................................. 30

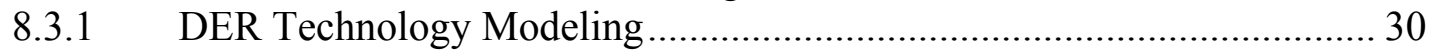

8.3.2 DER Dispatch Schedules ..................................................................... 31

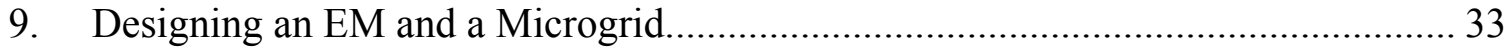

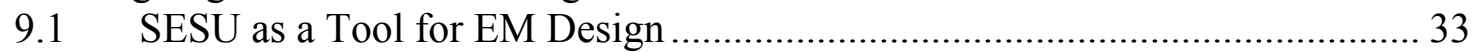

9.2 DER-CAM as a Tool for Microgrid Configuration Selection ......................... 33

10. Example EM Tests From Prior Case Study Work ............................................... 35

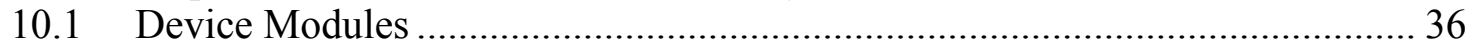

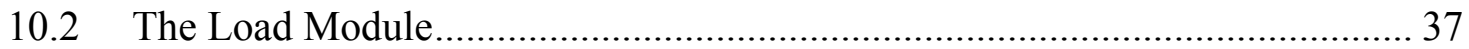

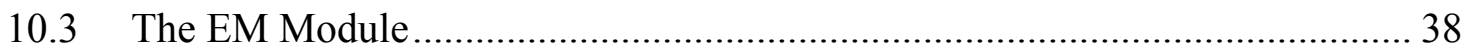

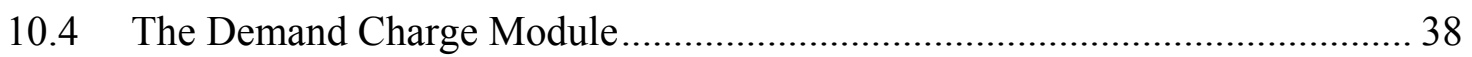

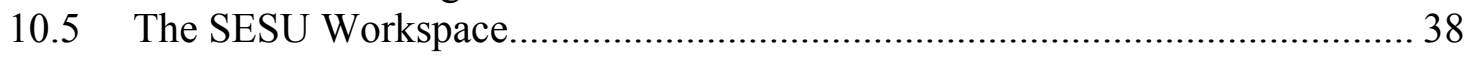

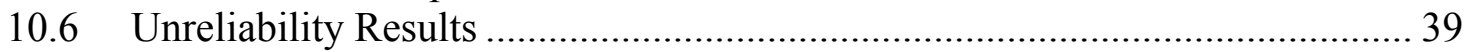

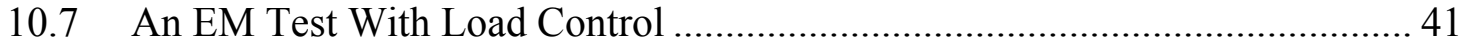

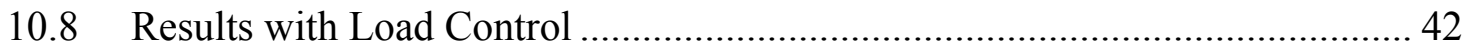

11. Dispatch for a Two Degree of Freedom Microgrid ............................................. 45

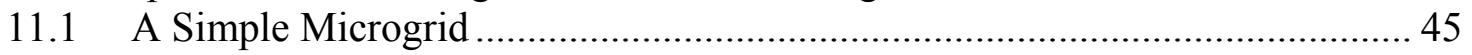

11.2 Optimization Strategy and Visualization ......................................................... 47

11.3 Scenario 1: No Heat Load and No Demand Charge Considerations ................. 48

11.4 Scenario 2: Heat Load and No Demand Charge Considerations ....................... 48

11.5 Scenario 3: Demand Charge Considerations but No Heat Load......................... 49

11.6 Scenario 4: Demand Charge Considerations and Heat Load.............................. 50

11.7 Optimal Control of Complex Microgrids ………......................................... 50

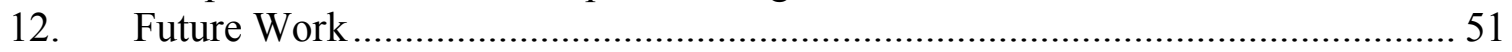

12.1 Costs of Acquiring, Transmitting, and Processing Data................................... 51

12.2 Modeling of DER Devices for Use in SESU ................................................... 51

12.3 Determination of Non-Critical Load Demand Control Costs ........................... 51

12.4 Control Strategies for Complex Systems ..................................................... 52

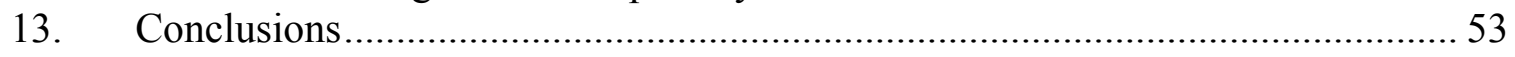

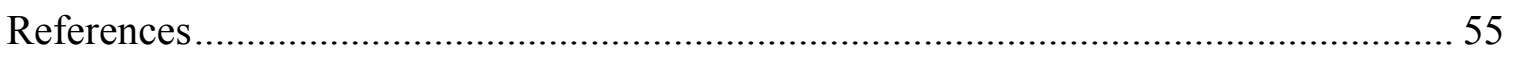

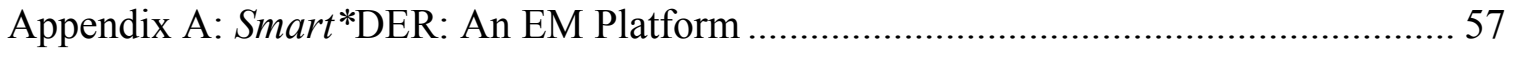


Energy Manager Design for Microgrids

\section{List of Tables}

Table 1: Natural Gas Engine Module Parameters............................................................... 36

Table 2: Expected Demand Charges.................................................................................... 40

Table 3: Demand Charge Variation Statistics.................................................................... 41

Table 4: Demand Charge Variation Statistics and Expected Value with Load Control .............. 43

Table 5: Microgrid Specifications ..................................................................................... 46

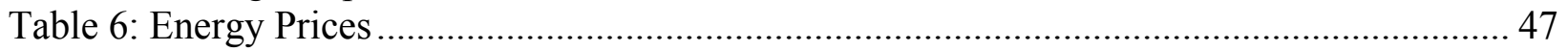




\section{List of Figures}

Figure 1: Probability Densities of Monthly Demand Charges for Generators 100\% Available (left) and 92\% Available (right)................................................................ $\mathrm{xv}$

Figure 2: Probability Density of Monthly Demand Charges for Generators $92 \%$ Available and Limited Load Control ........................................................................................... Xv

Figure 3: System Energy Cost Contour (\$/timeperiod) With Expected Maximum Utility

Demand Larger Than Current Load ............................................................................... xvi

Figure 4: System Energy Cost Contour (\$/timeperiod) With No Expected Utility Demand ...... xvi

Figure 5: Graphical Depiction of DER-CAM..................................................................... 30

Figure 6. Inside of Generator Bank Including Natural Gas Engine Modules................................ 36

Figure 7: Average Load Profile for June On-peak Hours.......................................................... 38

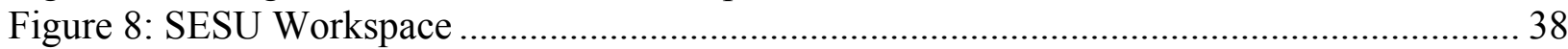

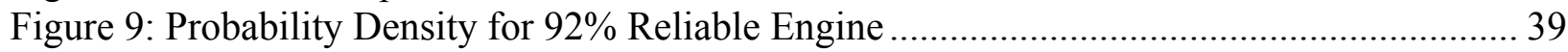

Figure 10: Probability Density for 96\% Reliable Engine …………............................................ 40

Figure 11: Probability Density for $100 \%$ Reliable Engine ......................................................... 40

Figure 12: SESU Workspace for microgrid with Load Control ............................................... 42

Figure 13: Probability Densities for Engine Reliability of 92\% (left) and 96\% (right) with

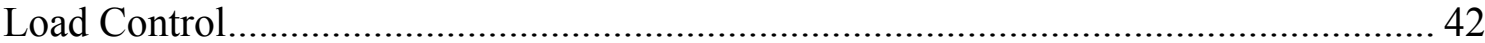

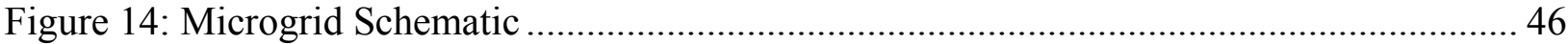

Figure 15: Scenario 1 System Energy Cost Contour (\$/hour) ...................................................... 48

Figure 16: Scenario 2 System Energy Cost Contour (\$/hour) ...................................................... 49

Figure 17: Scenario 3 System Energy Cost Contour (\$/hour) ...................................................... 49

Figure 18: Scenario 4 System Energy Cost Contour (\$/hour) ...................................................... 50 


\section{Acknowledgments}

We would like to thank program managers Mark Rawson, Bernard Treanton, and Laurie ten-Hope. Special thanks to Ron Hofmann and Joe Eto for their valuable comments on an early draft of this report.

This work builds on prior efforts supported by DOE through program manager Philip Overholt.

Finally, this work benefited from the work and insight of many current and prior members of the DER team at the Berkeley Lab. We would like to thank

- Owen Bailey

- Emily Bartholomew

- Norman Bourassa

- Charles Creighton
- Jennifer Edwards

- Kristina Hamachi LaCommare

- Bernard Lesieutre

- Tim Lipman
- Afzal Siddiqui

- Michael Stadler

- Nan Zhou 



\section{List of Acronyms, Abbreviations, and Definitions}

\begin{tabular}{|c|c|}
\hline CAISO & California Independent System Operator \\
\hline CERTS & Consortium for Electric Reliability Technology Solutions \\
\hline CHP & combined heat and power \\
\hline CPLEX & $\begin{array}{l}\text { numerical solver for linear programs, a trademark of CPLEX } \\
\text { Optimization, Inc. }\end{array}$ \\
\hline DER & distributed energy resources \\
\hline DER-CAM & Distributed Energy Resources Customer Adoption Model \\
\hline DOF & degrees of freedom \\
\hline EM & Energy Manager \\
\hline FERC & Federal Energy Regulatory Commission \\
\hline FIA & Facility Interface Agent in Smart*DER \\
\hline GAMS & $\begin{array}{l}\text { General Algebraic Modeling System, a trademark of the GAMS } \\
\text { Development Corporation }\end{array}$ \\
\hline GUI & graphical user interface \\
\hline HVAC & heating, ventilation, and air conditioning \\
\hline MATLAB & mathematical software, trademark of The MathWorks, Inc. \\
\hline OIA & owner interface agent in Smart*DER \\
\hline PRL & price-responsive load \\
\hline PURPA & Public Utility Regulatory Policy Act of 1978 \\
\hline QF & Qualifying Facility under PURPA \\
\hline RTP & real-time pricing \\
\hline SDG\&E & San Diego Gas and Electric \\
\hline SESU & Site Energy Supply and Use Model \\
\hline Smart*DER & a trademark of Alternative Energy Systems Consulting, Inc. \\
\hline $\begin{array}{l}\text { SPARK } \\
\text { TOU }\end{array}$ & $\begin{array}{l}\text { Simulation Problem Analysis and Research Kernel software } \\
\text { time of use rate schedule }\end{array}$ \\
\hline$\mu \mathrm{GRD}$ & $\begin{array}{l}\text { microgrid electrical modeling platform developed at the Georgia Institute } \\
\text { of Technology }\end{array}$ \\
\hline UPS & uninterruptible power supply \\
\hline
\end{tabular}





\section{Executive Summary}

On-site energy production, known as distributed energy resources (DER), offers consumers many benefits, such as bill savings and predictability, improved system efficiency, improved reliability, control over power quality, and in many cases, greener electricity. Additionally, DER systems can benefit electric utilities by reducing congestion on the grid, reducing the need for new generation and transmission capacity, and offering ancillary services such as voltage support and emergency demand response.

Local aggregations of distributed energy resources (DER) that may include active control of on-site end-use energy devices can be called microgrids. Microgrids require control to ensure safe operation and to make dispatch decisions that achieve system objectives such as cost minimization, reliability, efficiency and emissions requirements, while abiding by system constraints and regulatory rules. This control is performed by an energy manager (EM). Preferably, an EM will achieve operation reasonably close to the attainable optimum, it will do this by means robust to deviations from expected conditions, and it will not itself incur insupportable capital or operation and maintenance costs.

Also, microgrids can include supervision over end-uses, such as curtailing or rescheduling certain loads. By viewing a unified microgrid as a system of supply and demand, rather than simply a system of on-site generation devices, the benefits of integrated supply and demand control can be exploited, such as economic savings and improved system energy efficiency.

While the EM can provide supervision and suggest near-optimal operation of the microgrid, lower-level controllers, local to DER equipment and loads, may provide the actual control, upon receiving suggestions from the EM. This structure must facilitate interoperability of diverse devices and would allow for individual devices to veto suggestions from the EM that the device perceives as unacceptable or dangerous.

Pure optimal performance of a microgrid would require an all-knowing EM, which is clearly not practical. The set of information that affects microgrid performance must be examined to determine which information is most useful, which is obtainable, and at what cost. Naturally, intertwined with this analysis is the process of determining how the EM will make use of the acquired information.

The Consortium for Electric Reliability Technology Solutions (CERTS) Distributed Energy Resource Integration (DERI) team has developed a specific microgrid concept (the CERTS Microgrid) that relies on local power electronic electrical control and has modular, dynamic adaptability. However, such a dynamically reconfigurable microgrid places additional requirements on DER devices and on the EM.

Preferably, the system would be one to which components can be added or removed without the need for manual reconfiguration of the system. Dynamic adaptability is familiar in the field of computing, where components such as hard drives, keyboards, printers, and other accessories are readily integrated by the computer as long as the 
devices comply with known and supported standards. More recent standards require little action on the part of the user beyond physically plugging in the device, which is instantly recognized and operated. Beyond maintaining an updated registry of devices and loads on the microgrid and keeping the microgrid functional, dynamic adaptability here additionally implies the ability of the EM to maintain near-optimal supervision of the system as the registry changes. Such capabilities are highly desirable to the CERTS Microgrid.

Work within the CERTS DER team at the Ernest Orlando Lawrence Berkeley National Laboratory has led to development of software tools for microgrid design and operation. The DER Customer Adoption Model (DER-CAM) chooses economically optimal sets of DER devices to install for a given site. Additionally, the Site Energy Supply and Use Model (SESU) simulates operation of an installed microgrid and can be used as a testing ground for proposed EM strategies. These tools are of immediate use for assessing potential microgrids and to assist in the development of an EM. The authors expect microgrids to be installed in large numbers within a decade and the control approach embedded in them may endure for the foreseeable future.

To demonstrate how these tools can aid in microgrid development, examples provided in this report introduce some of the concepts of the EM and microgrid design, and they illustrate some issues that arise. The optimal constrained economic dispatch decision from an EM may be made over many discontinuous sets of options and may depend on past, present, and future conditions, suggesting overwhelming computation and data requirements. Therefore, the central question that must be addressed becomes what EM setup can deliver exactly the cost effective level of control; that is, one that meets all basic requirements and to which no added capability or data access adds a net energy bill savings, after the costs of implementation and operation are deducted.

In fact, making operational choices based purely on real-time conditions does not pose a significant challenge, the problem reduces to one of simply doing whatever is cheapest now. The much more troublesome complications the real world introduces are ones where non-deterministic future conditions affect current assessment of costs and where decisions affect multiple time periods. A consideration that has both proven to be critical in DER economics and decision making and demonstrates intertemporal effects is the ubiquitous demand charge in utility tariffs. Other considerations that require significant analysis include load scheduling and response to real-time stimuli, such as real-time pricing or emergency demand response programs.

Demand charges serve to illustrate several key EM issues in this report. One is that because of equipment unreliability, DER that reduces utility electricity purchase does not necessarily reduce demand charges. Figure 1 and Figure 2 present the probability densities of monthly demand charges for a modeled site with two on-site electricity generators and a stochastic load. Figure 1 (left) shows that if a relatively predictable demand charge results, varying only because of stochastic load (the installed DER is $100 \%$ reliable). If the DER is less than $100 \%$ available, outages occur and high demand charges result. Figure 1 (right) shows the probability density when both generators have 
a $92 \%$ availability. The two peaks going from left to right reflect the probabilities of both generators being available the entire month and that one generator is unavailable at some time during the month. The remaining demand charge occurrences to the right of the two humps result from both generators being unavailable simultaneously. Demand charges can be limited or reduced by simultaneously controlling the generators and loads. Figure 2 shows the probability density when the generators are $92 \%$ available and limited load curtailment is possible.
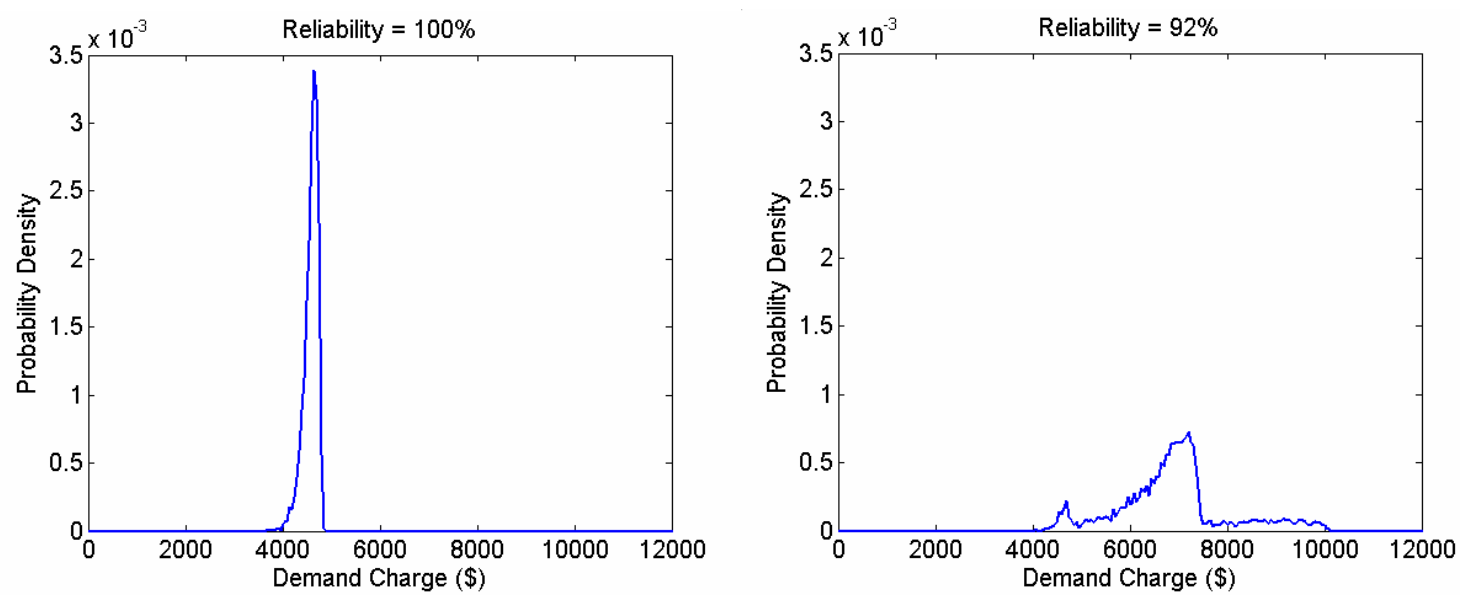

Figure 1: Probability Densities of Monthly Demand Charges for Generators 100\% Available (left) and 92\% Available (right)

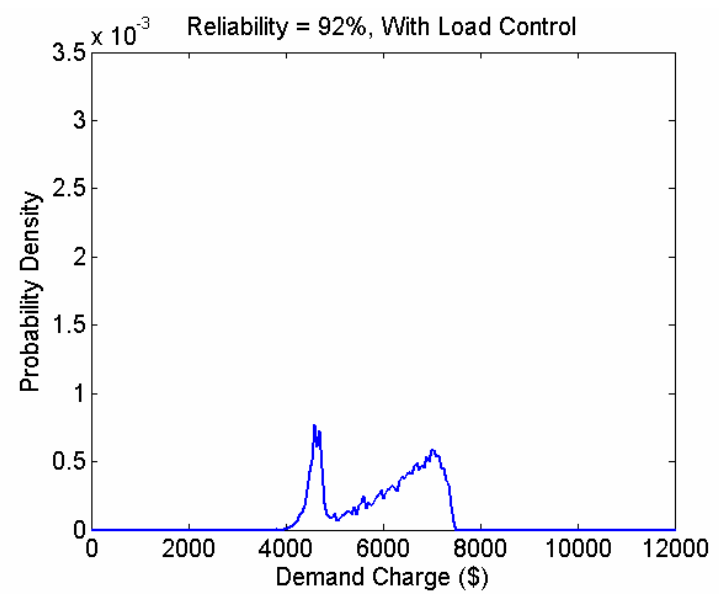

Figure 2: Probability Density of Monthly Demand Charges for Generators 92\% Available and Limited Load Control

Figure 1 shows the effect of availability on demand charge expected value and variance, while Figure 2 illustrates how load control that is responsive to DER equipment states can be used to reduce the expected value and variance of the demand charge.

Because demand charges are not determined until the end of the timeperiod (typically a month), real-time decisions are dependent on predictions of the future. A hypothetical microgrid with utility electricity service, a natural gas engine, and a microturbine is 
modeled, where the two generation devices can be either off or running from $50 \%$ to $100 \%$ of their rated capacity. Cost contours of the site's energy costs for the upcoming timeperiod show the EMs possible real-time dispatch decisions, and the subsequent system energy costs for that time period. Figure 3 shows the cost contour where no demand charges are considered because the EM predicts utility electricity consumption larger than the current electricity load later in the month. Figure 4 shows the cost contour when the EM predicts that the microgrid is capable of supplying all of the site's

electricity for the month, so that any dispatch that requires utility electricity purchase will result in demand charges, which are orders of magnitude larger than the other applicable grid electricity costs. The comparison of Figure 3 and Figure 4 illustrates that the optimal real-time decision depends not only on current conditions - which are the same in both scenarios - but also on predictions of future conditions.
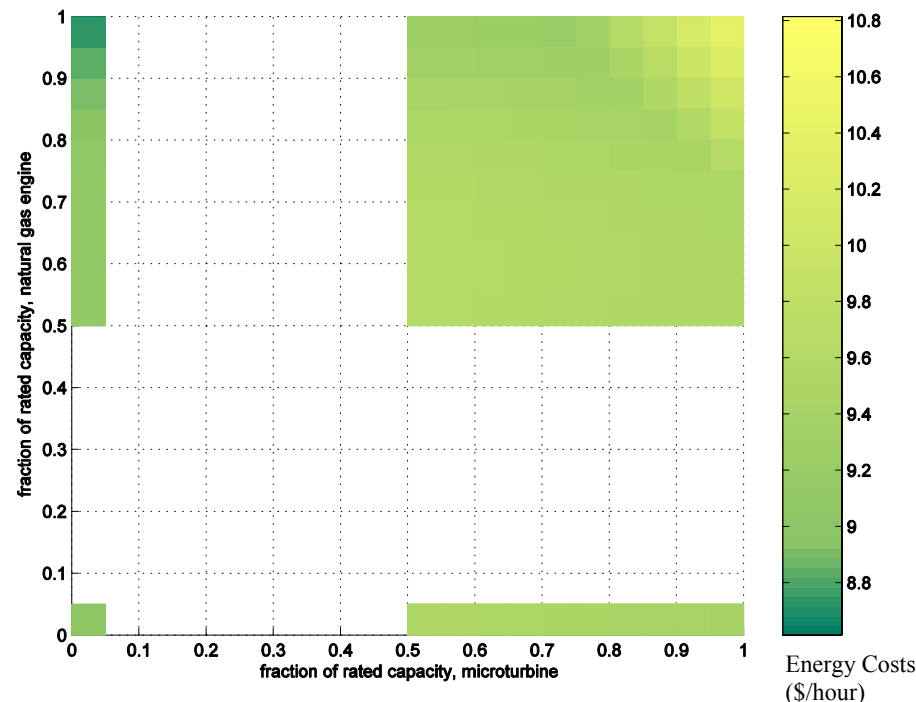

Figure 3: System Energy Cost Contour (\$/timeperiod) With Expected Maximum Utility Demand Larger Than Current Load

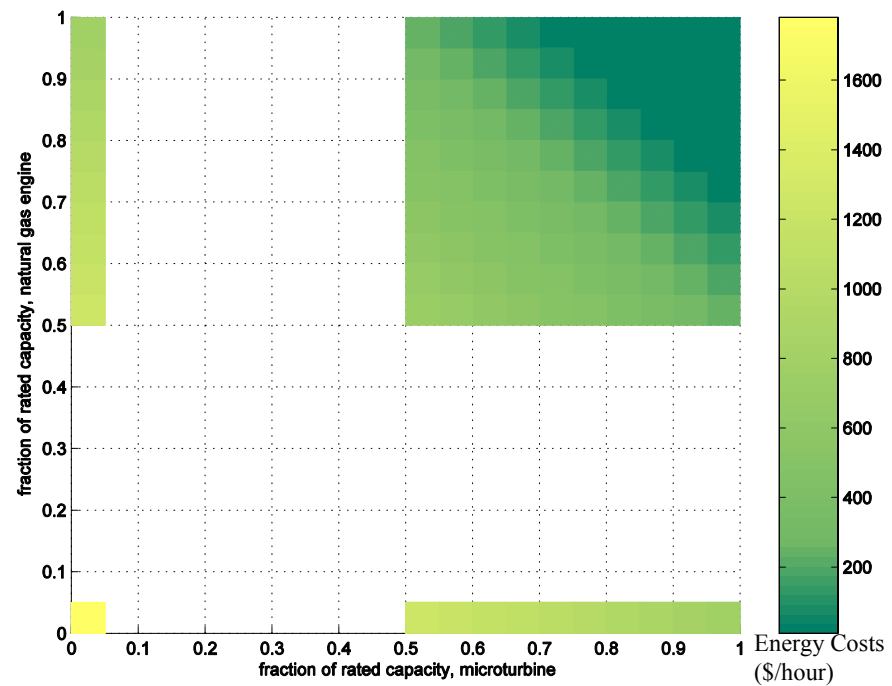

Figure 4: System Energy Cost Contour (\$/timeperiod) With No Expected Utility Demand 
Efficient EMs can minimize system operation costs, making microgrids attractive to the largest possible range of customers and ensuring that existing microgrids extract their maximum potential benefits. Research on EMs can reduce an individual microgrid site's costs to develop EMs, thus making microgrids more feasible and beneficial.

Generalizable results of EM research can improve the performance of microgrids, suggest equipment and microgrid design improvements, and aid in the development of effective policy to support the public's interests in DER. 



\section{Introduction}

Market deregulation continues to shape the United States energy sector. The Public Utility Regulatory Policy Act (PURPA) of 1978 first invited relatively small-scale generators into the electricity market, and wholesale competition has become widespread during the last decade, especially in some jurisdictions, notably California, New York, New England, and the Pennsylvania-New Jersey-Maryland interconnection. On the customer side of the meter, the change has been less dramatic, but nonetheless, expectations of the electricity supply system have evolved following experiments with customer choice, greater exposure of consumers to the variability of electricity prices, and an emerging generation roller coaster investment cycle. And, of course, California's market meltdown and associated reliability crisis, the terrorist threat, and the August 2003 Northeast blackout have radically reshaped expectations of supply security. Simultaneously, improvements to small scale and renewable technology have spurred an industry that has, in recent years, made even smaller (business scale) electricity generation an economically viable option for some consumers. On-site energy production, known as distributed energy resources (DER) offers consumers many benefits, such as bill savings and predictability, improved system efficiency, improved reliability, control over power quality, and in many cases, greener electricity. Additionally, DER systems can benefit electric utilities by reducing congestion on the grid, reducing the need for new generation and transmission capacity, and offering ancillary services such as voltage support and demand response.

Obviously, the regulatory framework will be a significant influence on the form of microgrids. The basic assumption adapted here is microgrids will operate under the electricity sector regulations of today and tomorrow; the focus of this work, however, is not on the regulatory regime but on the fundamental economic regime.

The most beneficial DER system for a given user might consist of multiple and varied electrical generation and storage devices, devices for heat recovery, thermal generation, energy storage, and devices such as absorption chillers and desiccant dehumidifiers that use heat to reduce electrical cooling loads. There may be multiple possible configurations for this equipment and it would not necessarily all be at the same physical location, e.g., heat sources might be placed near to the various heat loads. In addition, the most beneficial DER system might also interact with the energy demand of the site, curtailing or rescheduling certain loads. And naturally, the choice of systems should cooptimize equipment on the generation and end-use sides to minimize costs and maximize overall performance. Energy services from this system may also be provided to an aggregation of individual costumers in geographic proximity. This complex DER system is here referred to as a microgrid. This is the type of system to which this work is applicable. It is not directed at larger community scale or isolated power systems.

The Consortium for Electric Reliability Technology Solutions (CERTS) has developed the CERTS Microgrid concept, which is a particular microgrid paradigm whose electrical properties have been demonstrated in models (Lasseter, 2002). CERTS intends to conduct a laboratory test involving a few prototype devices in 2005, and to construct a 
functioning demonstration within the following two years. A key feature of the CERTS Microgrid is the separation of fast electrical control from the other slower control requirements to meet economic, heat load, environmental, and other constraints. An energy manager (EM) would perform these later functions.

A flexible, expandable system to which components can be added or removed without the need for extensive manual reconfiguration is highly desirable. Such capability is familiar in the field of computing, where components such as hard drives, keyboards, printers, and accessories are integrated by the computer. Restricting user requirements to little action beyond physically plugging in the device enables efficient, user-friendly configurable systems. As discussed in Chapter 2, these capabilities are highly desirable to the CERTS Microgrid, but such a flexible microgrid puts additional requirements on both the DER equipment and the EM.

\section{An EM is a "supervisory controller" that meets operational objectives and constraints} by dispatching devices. The development of an effective EM is one of the key tasks associated with the construction of a viable microgrid. Because of the complexities involved in the microgrid energy demands and supplies and the relative costs of using the microgrid versus purchasing from the utility, decision making requires information exchange and processing. Further, the EM must facilitate effective interoperability of heterogeneous devices. This report examines the processing requirements of the EM, the forms it might take, and the costs and benefits associated with various options.

Note that the specification for the EM above does not necessarily imply a specific physical device. The EM is more conceptual than functional, and its role could be performed in a variety of ways by a range of technical implementations from simple manual controls to highly sophisticated optimizing electronic controllers.

In contrast to the specific requirements for fast electrical control and protection management in the CERTS Microgrid, the functions of the EM are fairly generic and include many tasks that must be performed in any microgrid. Therefore, most discussion in this report will be generic in nature, and although this group of functions is referred to here as the EM in keeping with the CERTS paradigm and lexicon, many of the issues and principles discussed are relevant to other microgrid concepts. In an attempt to help the reader with this distinction, microgrid is here used to describe any semiautonomous Ogrouping of small-scale generation and load control, while CERTS Microgrid is used only for that specific paradigm, as described by Lasseter et. al. (Lasseter, 2002).

Work within the CERTS DER team at the Ernest Orlando Lawrence Berkeley National Laboratory has led to development of software tools for microgrid design and operation. The DER Customer Adoption Model (DER-CAM) chooses economically optimal sets of DER devices to install for a given site. Additionally, the Site Energy Supply and Use Model (SESU) simulates operation of an installed microgrid and can be used as a testing ground for proposed EM strategies. These tools are of immediate use for assessing potential microgrids and to assist in the development of an EM. The authors expect 
microgrids to be installed in large numbers within a decade and the control approach embedded in them may endure for the foreseeable future.

Chapter 2 discusses the CERTS Microgrid concept and the need for energy management. Chapter 3 discusses the information exchange in a microgrid that an EM might use. Chapter 4 examines control strategies. The concept of the dynamic adaptability is discussed in Chapter 5. Chapter 6 is an introduction to the second part of this report, which examines software tools developed at the Berkeley Lab and their use in developing optimal EMs and microgrid systems. Chapter 7 describes a software tool for modeling DER systems. Chapter 8 describes a software tool for selecting economically optimal DER systems. Chapter 9 proposes an algorithm for selecting an optimal microgrid using these tools. Chapter 10 offers an illustrative example. Chapter 11 describes some of the complexities in determining optimal dispatch. Chapter 12 describes some of the information gathering and technical work required to develop an actual EM. 



\section{The CERTS Microgrid Concept and the Need For an EM System}

The CERTS Microgrid consists of multiple devices that are viewed by the traditional power system, or macrogrid, as a single, controllable system. It may serve one customer or an aggregation of customers working in conjunction with traditional fuel and power suppliers.

CERTS Microgrid devices should have dynamically adaptable characteristics in order to overcome some of the barriers to DER adoption. Such microgrids would have many benefits over less modular designs, notably lower costs of DER system design and installation. Generation capacity and storage could be added or removed as microgrid technologies, demands, or economics changed over time. New technologies could be added to the system to increase capacity or to replace outdated equipment. Electricpowered vehicles, such as hybrid or fuel cell vehicles might be used for temporary on-site electricity generation when not in use (Lipman, 2002).

Development and implementation of an intelligent supervisorial system for DER equipment, such as the EM, will soon be necessary: CERTS intends to demonstrate its concept within the next two years. Conceptually, the EM can be expanded to simultaneously control building systems such as lighting, heating ventilation and air conditioning (HVAC), and/or industrial processes. The system will need to maintain functional, safe, and economic performance of the microgrid.

\subsection{Controls For the CERTS Microgrid}

To date, discussion of control for microgrids has focused on fast electrical considerations, but necessary controls for a CERTS Microgrid can be conceptually divided into three layers, the Microsource Controller, the Protection Coordinator, and the EM (Lasseter, 2002). The focus of this report is squarely on the third level.

\subsubsection{Microsource Controller}

In this discussion, a microsource is an on-site electrical generator such as a reciprocating engine, a microturbine, photovoltaics, or a fuel cell. The task of the Microsource Controller is to maintain the health, stability, and usefulness of the local electrical infrastructure while meeting utility interconnection requirements. This is achieved by locally controlling the phase, frequency, and voltage of electrical output from each electrical generation and storage device. Each device has its own independent power electronic controller that responds to events in milliseconds or tens of milliseconds.

\subsubsection{Protection Coordinator}

The Protection Coordinator would rapidly isolate feeder faults, allowing healthy sections of the CERTS Microgrid to continue operating during a fault, and islands the CERTS Microgrid from the utility during a fault to one or the other. Protective relays would need to be placed strategically throughout the grid. Controls necessary to trip these relays in 
the appropriate manner would be needed, in either a central or distributed manner, or some combination of the two (Feero, 2002).

\subsubsection{Energy Manager}

Finally, the function of interest here is the EM's task, which would be to approximately optimize use of individual DER devices. As mentioned above, this function is fairly generic and will be required in all microgrids. The objective will typically be to minimize the total energy bill within the constraints of the system, which might include serving heat and electrical loads, fuel costs, and equipment performance specifications, limitations due to safety, fuel supply limitations, and restrictions on noise or pollutant emissions.

The EM would supervise not only electrical microsources, but diverse DER devices such as end-use equipment, electrical, thermal, and mechanical energy storage devices, heat recovery and utilization equipment, absorption and adsorption cooling, desiccant dehumidification, etc. Optimization would most likely be to minimize cost but could also consider efficiency, emissions, system reliability, or other metrics of concern to individual microgrids. Unlike the microsource controller and the protection coordinator, which primarily concern energy supply of microgrids, the EM would be most effective by interacting with both the energy supply and demand. There would be one EM for the entire microgrid. It would generally make dispatch decisions in the order of minutes to hours but might respond more rapidly to certain stimuli such as grid emergencies. Lower-level controllers, such as the microsource controller, would be allowed to veto EM dispatches that were deemed unacceptable or dangerous.

The microgrid optimization problem is similar to traditional utility economic dispatch but differs in a few key ways. First, the central role of combined heat and power (CHP) applications in the microgrid requires joint optimization of both electrical and heat energy. Second, unlimited back-up purchase of grid electricity will normally be an option except during times of grid failure. Third, in contrast to utility scale systems that generally aim to maintain a minimum universal quality of service, the CERTS Microgrid specifically seeks to protect sensitive loads while potentially sacrificing or postponing others (possibly following an incoming demand response request), thereby providing a heterogeneous service standard.

The EM should always operate the microgrid in ways beneficial to the microgrid itself and at least be no more damaging than currently acceptable loads to the macrogrid, that is, it should be a good citizen of the grid. It should also be robust to extreme weather, fuel crises, macrogrid instability, and other threatening events.

\subsection{Design of the Energy Manager}

Candidate EMs must be able to control microgrid devices to meet any hard system restrictions, such as safety restrictions, operating bounds, e.g., minimum load levels, fuel limitations, and restrictions on noise and pollution. 
Candidate EMs should choose the minimum cost microgrid operation state within the subspace of possible microgrid operation states that meet these hard restrictions; EMs might make use of a wide variety of information and decision-making techniques to make this choice.

Evaluation of candidate EMs will depend on the desired definition of optimal performance. If the objective is energy cost minimization, then EMs can be compared by evaluating the energy costs, but this must include the cost of implementing the particular EM. In other words, a more costly EM must perform better.

The term energy management systems (EMS) is widely and inconsistently used, but systems relevant to the CERTS Microgrid have been discussed in prior work (Kueck, 2003). Kueck's EMS is assigned with the same tasks as the EM, namely to optimally dispatch DER equipment and flexible loads in response to energy demand, pricing, and operational constraints; however, it is more abstract than the EM concept described in this report, in that it does not consider the costs and limitations associated with access to information and information processing. In addition to selecting, defining, and designing the EM, it should be remembered that in most cases, a microgrid will be deployed in a facility with a pre-existing control system, such as a building control system. Achieving compatibility and interoperability with such systems poses major challenges that cannot be overlooked. Note that such building control systems are also frequently called energy management systems or energy management and control systems.

This report focuses more on the details of designing algorithms for an actual EM. As an introduction to some of the concepts involved in this design, an example that is useful in visualizing the optimal decision making an EM must make is provided (Section 1). 



\section{Information That Affects a Microgrid}

The set of all information that potentially affects microgrid operation is enormous and includes tariff details, equipment performance, weather conditions and forecasts, load details and forecasts, and thermal models of the microgrid. Some might be fairly easy and inexpensive to obtain while some would be prohibitively expensive or impossible to obtain, with a broad spectrum in-between. Absolute optimization of microgrid control would require all of this data; however, the costs of acquiring and processing it would be enormous or impossible.

Therefore, the set of information that affects the microgrid must be examined and important choices made. For each entry in this set, feasibility, procurement, and processing costs must be considered, as well as the alternative of approximating assumptions. All of this must be weighed against its usefulness to determine if it should be used by the microgrid control system. This chapter categorizes information that affects a microgrid and discusses its affect on the system.

\subsection{Tariff and Regulatory Information}

If total energy cost is to be minimized, then a comparison between energy costs from self-generation and utility purchase must be made; therefore current cost details of electricity, natural gas, propane, heating oil, and any other fuels must be known for operations.

Electricity purchase may take several forms. It could be purchased from traditional static utility tariffs with or without time-of-use (TOU) energy and demand charges, a dynamic utility tariff, such as a real-time pricing (RTP) scheme, or even directly from day-ahead or imbalance energy market such as ones run by the California Independent Operator (CAISO). Sites with their own generation capabilities and shedable loads are particularly suited to benefit from interruptible tariffs, emergency demand response incentives, or other schemes that lower the firmness of their demand.

In particular, demand charges add significant complexity to economic DER dispatch decisions, as the incremental cost of energy at a given time will not be known with certainty until the end of the demand charge period, typically a month. Additionally, interruptible tariffs create supply uncertainty. These issues illustrate that electricity costs are often not known in real time (in other words, the cost consequences of decisions made now are not fully knowable now), and highlight the need for prediction and planning, which dramatically complicates specification of control algorithms and increases the need for ex ante testing of their robustness.

In addition to tariff details, the EM must also be aware of and abide by regulatory constraints. Regulatory constraints may restrict the acceptable range of DER operations, and include restrictions on emissions and system efficiency. 


\subsection{Equipment Performance and Monitoring}

Each piece of DER equipment has a specified range of operating levels, and a particular level of performance at each operating level. This performance will depend on equipment capabilities as well as on external factors such as ambient temperature, humidity, and pressure. Performance might also depend on the age of the equipment and the time since the last maintenance. As microgrid loads will be constantly fluctuating, the dynamic properties (such as ramp rates) of the equipment might also be important considerations. Transients, such as start-up currents could even trigger significant costs if, for example demand charges are based on the average consumption over a brief timestep. The costs of maintaining equipment may vary with the amount or type of use that the equipment experiences.

This information could be gathered from manufacturers' specifications, by examination of performance data for currently operating equipment, or by analytical methods. Equipment can be monitored to detect or predict faults. Performance monitoring can additionally be used to refine the assumed equipment performance information.

DER operation may be constrained by on-site storage limits and/or by regulatory constraints. In such cases, dispatch decisions must weigh the benefit of operating equipment immediately against the benefits of operating equipment at a later time or reserving operation capabilities for back-up power during grid outages. These constraints are often encountered with diesel engines, which are typically limited both by air quality permit restrictions and by the available on-site fuel storage capacity and replenishment cycle. More generally, capacity constraints apply to any on-site energy storage, including thermal and electrical.

\subsection{Atmospheric Predictions and Data}

Weather conditions will affect the performance of DER equipment itself, change the sensitivity of the local environment to DER emissions, and more importantly significantly affect the energy demand of a site. Real-time monitoring of weather conditions can be used in conjunction with long-term forecasting (based on historical weather patterns) and short-term forecasting (based on weather forecasts for the coming hour, day, week, or season).

Historical weather data are readily available, and average historical patterns, as well as a statistical description of the data can be readily derived. Real-time weather monitoring might entail reading data from sensors at the site or downloading regional data from a weather service.

\subsection{Load Prediction and System Models}

Energy loads can be categorized by their end-use. DER studies at Berkeley Lab have focused on the following five end-use load types intended to distinguish loads that are CHP candidates from those that are not: electricity-only (excludes cooling), cooling and refrigeration, space heating, water heating, and loads that can only be met by combustible 
fuel (such as natural-gas cooking). Other load types might be appropriate to particular microgrids, and include process heating and mechanical-work loads.

The EM needs to know the real-time energy load requirements of the microgrid in order to assure that these requirements are met. Furthermore, an EM may use planning in its control if the load data for a site can be predicted based on historical load data and knowledge of how the current system and conditions will alter the loads from their historical values. Historical load data are more accurately described statistically than as averages.

While electrical loads must be met instantaneously, thermal loads can be met more flexibly. Heating and cooling systems store energy in operating fluids, thermal loads can be met for a time even if no additional energy is delivered to the boiler or chiller. Additionally, the mass of the structure being heated or cooled provides thermal lag so that temperatures change only gradually when heating or cooling stops. Additional thermal storage can be added to energy systems to provide further operating flexibility. EMs can make use of system thermal models and indoor climate constraints to determine the extent of this flexibility and the allowable range of dispatch to thermal systems.

\subsection{Heterogeneous Service}

A key element of the CERTS Microgrid is the aim to provide a heterogeneous quality of service within the microgrid, in contrast to the universal service provided by the macrogrid. Load control in this context can be thought of as heterogeneous reliability because some of the end-uses are being assigned a higher or lower priority on limited resources. If the quality of service is to be controlled, then first it needs to be categorized and parameterized. Most simply, on-site end-uses might be divided into three categories: critical, curtailable, and reschedulable.

More generally, demand control would be attractive to a microgrid because better performance can be achieved if an EM can control both its supply and its demand. However, there would be incurred costs in implementing integrated controls. One situation where demand control is economically attractive might be when dealing with extremely peaky energy prices, which can come from demand charges, TOU rates, market, or RTP price spikes. Another may be an emergency demand response incentive. If demand can be adjusted to be less peaky, a system with smaller capacity will minimize costs, often with less reliance on the macrogrid. In other words, flattening the load both lowers the peak import demand and the necessary on-site capacity required to meet the peak; therefore, the optimal generating capacity will be smaller. For this reason, effective implementation of CHP driven cooling is a particularly potent source of bill savings because it lowers loads at likely peak times, i.e., potentially very expensive, times.

In cogeneration applications, demand management can be used to more closely match supply and demand of the different energy types, for example scheduling processes that require heat at times when a microsource will be running to meet the electrical requirement and significant waste heat will be available. 


\subsubsection{Critical Demand}

Critical demand describes loads that must be met at all times, such as servers and loads related to essential processes. Note that if a microgrid does not have special demand control capabilities, then all loads are viewed as critical loads. A microgrid must meet all critical loads in preference to any other load, and possibly without regard to cost. Special equipment, such as uninterruptible power supplies (UPS), are commonly currently found fulfilling this function on a small scale or temporarily. Critical loads may be prioritized in the event that load shedding is required due to supply limits.

\subsubsection{Curtailable Demand}

The magnitude of certain demands might be flexible. Curtailable demands have a preferred level, but the demand level can be lowered if a certain cost is associated with the load reduction. For example, air conditioning operates at a temperature setpoint requiring a certain chiller load. If the setpoint is raised, a warmer indoor building temperature ensues and lowers occupant amenity. During times of particularly high electricity prices, the cost of this incremental discomfort may be outweighed by the incremental cost of electricity purchase.

Curtailable loads could be specified by the following parameters:

- full load demand,

- percentage of load that can be curtailed,

- cost of curtailment,

- lead time needed before curtailment can begin,

- ramp rate at which load can go down,

- length of time for which load can be curtailed, and

- maximum frequency of curtailments.

Putting an accurate monetary price on the cost of curtailment will be difficult in most cases. However, the savings from load curtailment during short periods of high electricity pricing or microsource outages may justify examining this issue.

\subsubsection{Reschedulable Demand}

Certain demands may be flexible in their scheduling. Rescheduling might mean staggering the start of several electric motors so that the large current associated with start-up is broken into several moderate power spikes rather than one large one. This would be useful in avoiding demand charges or overloading an isolated microgrid. Rescheduling could also involve shifting the execution of some energy intensive activities to later in the day, or further into the future.

If the EM is creating a schedule that goes into the future, rather than just handling realtime energy requests, then loads can be rescheduled backwards in time. An example of this would be pre-cooling a building during the less energy intensive (and less expensive) hours of the morning rather than waiting to start cooling until there is a cooling demand. 
Reschedulable loads could be specified by the following parameters:

- load demand,

- maximum length of rescheduling time, or acceptable time to reschedule,

- cost of rescheduling,

- lead time needed before rescheduling can take effect, and

- rate at which load goes down once it is rescheduled.

Demand control could add a significant level of complexity to an EM. Whereas loads are traditionally allowed to be raised to any level at any time, loads would now have to request energy, and only draw energy when and to the extent allowed by the EM. This would require enabled loads to communicate their status to the EM and to be intelligent enough to curtail or reschedule themselves safely. Demand control is already being implemented in some commercial building EMS ${ }^{1,2}$. Some demand control might only be semi-autonomous, with significant rescheduling or curtailment requiring the approval of a building manager or other person in charge of the microgrid, and override capability will always be necessary.

\footnotetext{
${ }^{1}$ Appendix A of Kueck (2003) mentions several systems currently available for automation of building load control.

${ }^{2}$ For example, PowerLight, a Berkeley, CA based photovoltaic system provider has partnered with CMS Viron Energy Services, the energy performance contracting unit of CMS Energy Corporation, to provide building energy systems that automatically reduce non-critical loads during temporary reductions in photovoltaic output due to cloud cover. http://www.escperform.org/stories/alameda.htm
} 



\section{Control Strategies Available for Microgrids}

Along with the set of input information, the CERTS Microgrid will need a strategy for making decisions. For a given set of information that an EM has access to, there are many control strategies that could be employed. Each must be judged by the microgrid performance it achieves and the costs associated with its implementation. Three possible control strategies are real-time optimization, expert system control, and decentralized control.

\subsection{Real-time Optimization}

Real-time optimization is the most computationally intensive control strategy available to EMs. EMs would first consider the past and current microgrid operation states, loads, weather, tariffs, and equipment, then consider the stochastic description of loads, weather, tariffs, and equipment in order to predict future microgrid operation states, and then dispatch an operation decision to the microgrid equipment. While this is not realistically possible at present, approximations of this optimization would be. Certain information could be approximated by constant values that describe the system reasonably well and do not lead to large deviations from actual behavior.

Much of the field of operations research focuses on constrained optimization problems. A system of consideration is described mathematically as an objective function to be maximized or minimized, and constraints on variables. The problem is then to determine the state (a set of allowable variable values) that results in the maximum or minimum objective function.

Many algorithms and software are available to solve such optimization problems. The power of these algorithms lies in intelligence replacing the brute force of enumeration, i.e., rather than evaluating the objective function at every allowable state in order to determine the optimal state, intelligent searches exploit their knowledge of the structure of a system in order to only consider a small fraction of allowable states.

If systems can be approximated to fit into certain mathematical structures, specific theory and algorithms can be applied (Hillier, 2001). The fastest and most certain results come from systems that can be described as linear programs. The objective function and constraints of a linear program must all be linear equations. More complex are linear systems with integer constraints ${ }^{3}$. Nonlinear systems add additional complexity. Algorithms for nonlinear systems have been tailored to systems that can be described as

\footnotetext{
${ }^{3}$ Integer constraints include yes/no decisions, machines, people, or other objects that can only exist in integer quantities, and machines with a finite number of setpoints.
} 
quadratic objective functions ${ }^{4}$ with linear constraints, separable systems, ${ }^{5}$ or convex systems. ${ }^{6}$ Algorithms for more general non-linear programs also exist.

Microgrid systems contain many program complexities such as non-linearities in part load equipment performance, non-linearities in tariff structures (such as demand charges), integer decisions in turning equipment on or off, uncertainty about the future, rescheduling options, equipment start up times and costs, and equipment ramp-rates, to name a few. For a microgrid real-time optimization, the EM must construct a mathematical description of its optimization problem. Depending on the level of system detail desired for the optimization, the computing power of the EM, and time constraints, the EM can use approximations to reduce the complexity of the model to a feasibly tractable system and use optimization algorithms or commercial software to select an optimal dispatch state and scheduling detail. The example in Section 1 illustrates several of the considerations involved in this type of control strategy.

\subsection{Expert Systems and Fuzzy Expert Systems}

Absolute optimization, or an approximation of it, may be too computationally taxing or expensive in real time. It may be more realistic to have an EM that has a finite set of control options and simply assesses which option to apply given the past, current, and predicted future states of the system. This rule-based technique is known as expert system control.

An expert system control approach to microgrids would consist of the EM assessing the state of the microgrid (demand levels, load control options, supply options, costs, weather, etc.), determining what predefined category the current state falls into, and following the dispatch rule associated with that category. This type of control could be programmed as a series of If-Then statements.

Unlike a real-time optimization, optimization in expert system control is done prior to EM implementation, in determining categories and rules. Adaptive control strategies could redefine categories or rules as the system is in operation based on system performance.

It is not obvious how to categorize the states of a microgrid, or how to determine optimal dispatch for each category. One benefit of expert system control is that it mirrors human reasoning. First generation controllers can be developed quickly by studying human operators of energy systems and mimicking their process of categorizing system states and applying rules to specific categories. Modeling of the DER system is required only to the extent to which the human operators use knowledge of the system to make

\footnotetext{
${ }^{4}$ Quadric equations consist of a sum of terms in which variables appear in terms as squares $\left(\mathrm{x}^{2}\right)$ or products of two different variables $\left(\mathrm{x}_{1} \mathrm{x}_{2}\right)$

${ }^{5}$ In separable systems, equations and inequalities are the sum of individual terms that are functions of only one variable per term ( $\operatorname{such}$ as $2 \mathrm{x}, \mathrm{x}^{3}, 12 / \mathrm{x}$, but not $\mathrm{x}_{1} \mathrm{x}_{2}$ )

${ }^{6}$ In convex systems, equations and inequalities are the sum of individual terms that are convex functions. Convex functions are those that always curve upward (positive second derivative)- i.e. a local minimum is guaranteed to be the global minimum. Linear functions are also convex functions.
} 
decisions. Experimentation with state categorization and response can then lead to improved expert system controllers.

Another way to develop these If-Then rules would be to use a model of the DER system. Analysis could be performed using the model by experimenting with different control schemes or implementing a real-time optimization on the simulated system. Optimal control could be determined on this model. Generalizations could then be made by arranging the set of past, present, and predicted future states of the systems into a finite set of categories. Then, the determined control for the set of states within a particular category could be generalized into one dispatch scheme.

Fuzzy expert systems are an extension of expert systems (McNeil, 1993). Fuzzy expert systems consider overlapping categories of states, and assess to what extent the system is in each particular category. Here, several "If" conditions are satisfied simultaneously (yet to different extents), and the subsequent control dispatch is a weighted combination of the respective "Then" statements. "Fuzzification" allows for more complex rule-based control than traditional expert system control.

\subsection{Decentralized and Hierarchical Control}

Control strategies could be developed in which individual devices act as agents that can communicate with other device agents in order to make decisions. Although not directly examined here, this strategy has been suggested to aggregate individual microgrids to bid their excess capacity to the grid (Gibson, 2001). In this same manner, an aggregation of individual energy suppliers (i.e., microsources in a microgrid) could bid their capacity to the loads of the microgrid.

The decision making in decentralized control must still have a hierarchical structure. A single agency would collect demand and supply bids from multiple agents and follow established rules for making dispatch decisions for individual agents. These rules that the agencies abide by may be determined by an even higher-level controller.

This describes the hierarchical control of utility grids. Similar to the microgrid, individual electricity consumers and producers are agents. These agents report to a system operator that determines dispatch based on these reports and rules provided by governing authorities.

As with expert system controllers, decentralized, hierarchical control achieves optimization through the design of the bidding behavior and dispatch rules, rather than exhaustive real-time calculations. It also lends itself well to dynamically configured systems (see Chapter 5) because the EM itself does not need to update its perception of the microgrid, it only needs to accept bids (from energy supply devices or utilities) and respect end-use load requests in order to make dispatch decisions. 



\section{The Dynamically Adaptable Microgrid}

A dynamically adaptable system is one to which, over time, components can be added or removed without the need for extensive manual reconfiguration of the system. As discussed in Chapter 2, such capabilities are highly desirable to the CERTS Microgrid, but such a flexible microgrid puts additional requirements on both DER equipment and the EM.

The computer version of dynamic adaptability arose from developments by Mircrosoft in 1995. In this scheme, upon booting a computer, the basic input/output system (BIOS), which is software installed on central system hardware, begins by polling the devices connected to the system hardware and comparing this to the last known system configuration. Devices must have special capabilities in order for them to let the BIOS identify them. If the system configuration has not changed, the bootup process continues. Otherwise, the BIOS creates a new system configuration and description for the operating system and stores it in the extended system configuration data (ESCD). After bootup, the operating system (OS) can then use the ESCD to identify what devices are connected where and thus interpret incoming data correctly and make decisions accordingly about resource allocation and data output (Kozierok, 2003). Modern computers allow devices to be hot switched while running and for multiple devices to be connected through one port.

However, note that dynamic adaptability does not imply that one consistent standard must be maintained, just as PCs have employed multiple standards together and over time such as serial and parallel connections, USB and firewire.

\subsection{Requirements on DER Equipment for a Dynamically Adaptable Microgrid}

Each controlled device that is added to a microgrid must be responsible for letting the EM know what the device does and what its performance parameters are. Devices can be categorized into classes and the EM would need to know the characteristics of devices in each class. The devices and EM, in this sense, are analogous to the devices and BIOS in the computer scheme. The EM further serves the function of the OS, interpreting incoming signals to determine output dispatch.

One category of devices might be electrical generators. The EM would know a priori that generators require fuel and produce electricity and waste heat. Then, a specific device such as a microturbine would be responsible for telling the energy manger that it is an electrical generator and that it consumes natural gas. Additionally, the microturbine would specify parameters about itself: its efficiency at varying load levels, its heat output (type of heat fluid such as exhaust or radiator fluid loop, temperature, and pressure at varying loads), its operation and maintenance costs, emissions, noise, etc. A diesel engine would fall in this same category and could distinguish itself from the microturbine by its different set of parameters. Other device categories might be photovoltaics, solar thermal devices, electrical storage devices, thermal storage devices, and chillers.

The most reasonable way for equipment to inform the EM about its characteristics is by two-way communication. Protocol for stating DER class and performance characteristics 
would need to be developed. Information objects containing this information could be transferred over a communication network already in place for devices to report their status to the EM and to receive control orders from the EM. Over time and the range of DER equipment and loads in communication with the EM, interoperability, the support of multiple object formats, will be needed.

\subsection{Requirements on the EM for a Dynamically Adaptable Microgrid}

The dynamically adaptable EM must be able to maintain a registry of active energy devices and loads on the microgrid. Additionally, the EM must contain the analytical ability to assess the updated microgrid and determine near-optimal dispatch of DER equipment, as well as curtailable and reschedulable loads.

\subsubsection{Supply Side: DER Equipment}

An evolving microgrid would require an EM be flexible enough to make decisions for configurations that the owners choose to install in the future. In order to do this, it must know the set of DER categories that equipment can fall under and how to interpret the performance parameters within each category. The EM must be able to receive this information from the DER equipment and it must be able to modify its data description perception of the microgrid as components are added or removed. In practice, an upgrade in the field may be used to accomplish this. For example, an additional plug-in EM module might accompany a new device, or a downloadable software upgrade might accomplish the reconfiguration.

\subsubsection{Demand Side}

For demand control, energy users (such as machinery or heat loops) must be able to communicate their status as critical, curtailable, or reschedulable load, as well as their respective parameters as identified in Section 3.5.

\subsection{A Microgrid Without an EM}

As the reader will have discerned by now, the EM is not readily defined. It is more conceptual than physical, more operational than programmable. And, in fact, the functions described heretofore could well be most efficiently performed without need of a central physical device in some instances. Although requiring more manual intervention, it is possible to have a microgrid without an automated EM. Timers could be used to turn DER devices on and off at pre-determined times. Microsources could be set to run at a specific capacity or could be set to follow loads. Some amount of control optimization could even be performed if each device acted as an agent in communication with other devices (Section 4.3).

The lack of a central EM would have its consequences. There would be nothing to enforce restrictions unless individual devices knew about restrictions and were intelligent enough to operate within acceptable ranges. Also, functioning efficiently when some equipment is unavailable would present a major challenge. Again, the difficulty of 
meeting global constraints poses obvious problems absent a central EM. One example where a global constraint might be a problem is when a microgrid is required to meet the Federal Energy Regulatory Commission (FERC) approved qualifying facility (QF) efficiency requirement ${ }^{7}$. QFs may receive incentives such as special tariff structures or the option to sell excess electrical capacity back to the grid. A requirement of a QF is that they be $42.5 \%$ efficient as calculated by FERC's QF cogeneration efficiency calculation. Generally, this means that some amount of waste heat from electrical generation must be utilized. Without an EM, devices might run at many times when there is no need for waste heat utilization, thus lowering the overall efficiency. If the efficiency ended up being less than $42.5 \%$ on an annual basis, the site could forfeit benefits. An EM could globally control operation such that the efficiency stayed at $42.5 \%$ or above.

\footnotetext{
${ }^{7}$ Code of Federal Regulations, document 18CFR292.205 requires natural gas or oil fueled cogeneration facilities to have an overall energy efficiency greater than $42.5 \%$. Energy efficiency is defined as the percentage of fuel energy input that is converted to electrical energy output plus one half of the percentage of fuel energy input that is converted to useful thermal energy output.
} 



\section{Optimal EM Development}

Chapters 2 through 5 discussed the need for an EM, its required tasks, and the possible forms it could take. The Berkeley Lab has developed a model for optimal DER system selection and is developing a modeling platform for a more detailed assessment of a specific DER system under the control of a specific EM. These tools can be used to select candidate DER systems and to design and evaluate candidate EMs for a given DER system.

Chapter 7 discusses the Site Energy Supply and Use Model (SESU) software under development and its usefulness in evaluating candidate EMs. Chapter 8 discusses the DER Customer Adoption Model (DER-CAM) software and its usefulness in selecting candidate DER systems. Chapter 9 proposes an algorithm for designing a microgrid, complete with EM, using DER-CAM and SESU. This algorithm could be used to develop a CERTS demonstration Microgrid. 



\section{The Site Energy Supply and Use Model}

Assessment of candidate EMs requires a model of the microgrid under consideration. Modeling for microgrids for this purpose should capture the relevant details of the physical system yet must be convenient to develop in order to be useful as a tool in DER system design.

The Site Energy Supply and Use Model (SESU) is a MATLAB/Simulink DER system modeling tool in development at the Berkeley Lab. SESU allows users to draw block diagrams representing a microgrid. These block diagrams are then evaluated over the time period of interest to the user in order to determine the performance of the DER in terms of energy costs or system efficiency.

SESU will be used in the development of the CERTS Microgrid field demonstration to assess the performance of the microgrid under the control of candidate EMs. The most favorable candidate will be chosen by estimating the performance of the microgrid before construction.

\subsection{MATLAB and Simulink ${ }^{8}$}

MATLAB integrates mathematical computing, visualization, and a powerful programming language to provide a flexible environment for technical computing. Simulink is an interactive tool for modeling, simulating, and analyzing dynamic systems. Programming in Simulink is done by building block diagrams. MATLAB code can be introduced into Simulink modules to describe the dynamics of the module, as systems of continuous time differential equations, discrete time systems, or a combination of the two. For reasons of computational practicality, SESU modules will be limited to discrete time systems with timesteps in the range of minutes.

A Simulink device module can be developed to represent any possible DER technology. The heart of each module is MATLAB code that describes either the discrete time and/or continuous time dynamics of a class of devices, such as microturbines, heat exchangers, or boilers. Also contained in each module are the parameters specific to a member of the device class. An example of this might be a $30 \mathrm{~kW}$ microturbine module, which would contain the microturbine class MATLAB code and the parameters of that particular microturbine, such as its rated capacity, efficiency at varying loads, and heat output characteristics.

The high level programming language of MATLAB, together with its data processing and visualization capabilities, permitted rapid development of SESU. Simulink performs the dual tasks of being a user friendly, graphical environment, and determining a comprehensive system description based on individual modules and their connections.

\footnotetext{
${ }^{8}$ MATLAB and Simulink are registered trademarks of The MathWorks, Inc.
} 


\subsection{SESU Modules}

SESU modules are differentiated by their function. Together, the various types of modules can describe a complete DER system. The three main module types in SESU are device modules, load modules, and the EM module.

\subsubsection{Device Modules}

Device modules for specific DER devices can be selected and "drag-and-dropped" into a visual microgrid workspace using Simulink's graphical user interface (GUI). Each device module has input and output ports for physical connections such as heat loops, heat loads, and electrical loads. An additional input port accepts a control signal from the EM module, and additional output ports may return device identification, parameters, and performance information.

\subsubsection{Load Modules}

Load modules are specific to the energy type and status as critical, curtailable, or reschedulable. If no demand control is incorporated in the microgrid, then all loads can be described within critical load modules. If demand control is incorporated, loads need to be separated by type and pertinent parameters as discussed in Section 3.5.

\subsubsection{The EM Module}

Control of the system is determined by the EM module. The EM module has input ports to receive device, load, and other system data, and output ports to send control signals to devices and non-critical loads. Inside of the EM module, MATLAB codes needs to be developed to execute the algorithms of a particular EM scheme. For dynamically adaptable systems, EMs will need to be flexible enough to alter their perception and control of the system as the system changes.

\subsection{Using SESU}

The device, load, and EM modules are all placed graphically in a Simulink workspace. Connections between modules are made by clicking and dragging from output ports to input ports. In this way, electrical sources can be connected to electrical loads, heat sources to heat sinks or loops, and the communication network between devices and the EM can be established.

After a model is fully described, Simulink compiles the model to generate one system, rather than separate modules. The user specifies the desired length of time to examine the model behavior over (most likely hours to years) and Simulink determines the behavior of the system over time, including accumulated energy costs, device performance, and emissions statistics.

Chapter 10 illustrates the procedure of modeling a microgrid in SESU and assessing candidate EMs. 


\subsection{Related Work in the Modular Modeling of Configurable Systems}

The modular approach to modeling of configurable systems is common in the energy industry. Two software packages developed by our colleagues that offer promise for integration with SESU are $\mu$ GRD and SPARK ${ }^{9}$.

\subsection{1 $\mu \mathrm{GRD}$}

The $\mu \mathrm{GRD}$ analysis program is a software package developed at the Georgia Institute of Technology in which a microgrid's electrical system can be graphically described to determine the steady state power flows of the system. This program is useful in determining the transmission line loads, line losses, power quality, and electrical system stability for a given microgrid. Incorporating the electricity distribution modeling capabilities and power-flow analysis of $\mu$ GRD into SESU would allow SESU to determine system performance from both its current higher-level perspective and the lower-level perspective of $\mu \mathrm{GRD}$.

\subsubsection{SPARK}

The Simulation Problem Analysis and Research Kernel (SPARK) is a software system developed at the Berkeley Labs in which models are made by describing the dynamics of individual modules and then describing the interconnection of individual modules. While the modeling capabilities generalize to any system, they have been tailored, to date, for use on HVAC system modeling and have been combined with EnergyPlus ${ }^{10}$ for more accurate HVAC detail in building energy system modeling.

The SESU modules could well be described in SPARK. Combined with the marriage of EnergyPlus and SPARK that is on-going, this would enable users to model an entire microgrid, including the buildings that are served by the microgrid. This would allow for building models to replace the current heating and cooling load approximations used in SESU and DER-CAM modeling. A graphical editor for programming in SPARK has yet to be developed.

\footnotetext{
${ }^{9}$ SPARK documentation and software downloads are available at http://simulationresearch.lbl.gov/

${ }^{10}$ EnergyPlus is building energy simulation program developed jointly by Lawrence Berkeley National Laboratory, the University of Illinois, the U.S. Army Construction Engineering Research Laboratory, GARD Analytics, Inc., Oklahoma State University and others, with support from the U.S. Department of Energy, Office of Building Technology, State and Community Programs. EnergyPlus is a trademark of the U.S. Department of Energy, Dru Crawley, Program Manager. Documentation can be found at http://simulationresearch.lbl.gov/
} 



\section{DER-CAM}

DER-CAM is a software tool developed at the Berkeley Lab in which the economically optimal CHP DER system is determined for a site, given the site's energy usage, utility tariffs, and DER equipment options.

If a candidate CERTS Microgrid demonstration site installs DER equipment, DER-CAM could be used to select candidate DER systems. SESU could later be used to assess these candidate systems in more detail, under actual control strategies.

\subsection{DER-CAM Modeling and Results}

Typical inputs to DER-CAM include the site's five load profiles, tariff structure under which the site buys electricity and other fuels, and values from a database of technology costs and performance. The five load profiles are electricity-only (not including cooling), cooling and refrigeration, space heating, water heating, and natural gas only. The output is a set of installed DER technologies that minimize annual costs of meeting energy demand for the site, an hourly operating schedule of each selected technology, and utility energy purchases.

The objective function to be minimized is the annual cost of providing energy services to the site, through either utility electricity and gas purchases, or DER operation, or a combination of both. This value is a summation of costs for electricity purchases, gas purchases, capitalized costs of DER equipment, and operating and maintenance costs.

A key constraint is that energy demand for each hour must be met by the purchase of energy from utilities, operation of any technology or set of technologies selected by the model, or a combination of purchase and on-site generation. In addition, specified environmental rules must be obeyed, and equipment capabilities must not be exceeded.

The output of DER-CAM includes the economically optimal set of technologies to install, a dispatch schedule for that year, and the annual energy costs.

The model's inputs and outputs are depicted graphically in Figure 5. 


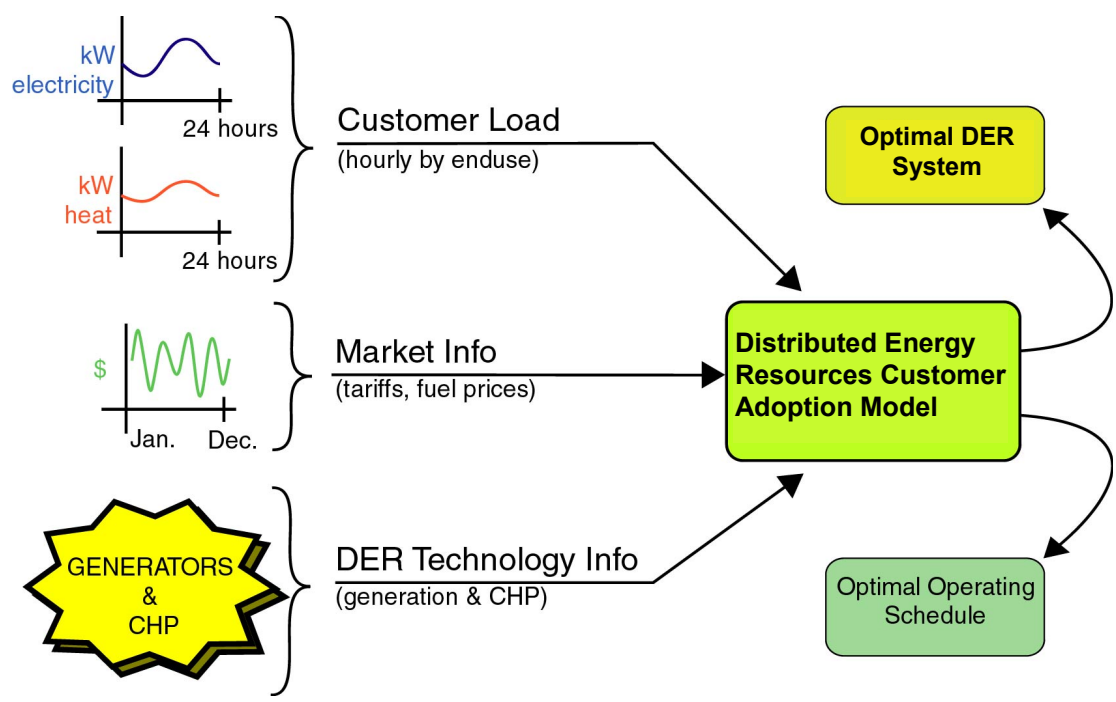

Figure 5: Graphical Depiction of DER-CAM

DER-CAM is a cost minimization mixed integer program formulated in GAMS ${ }^{11}$ (General Algebraic Modeling System) and solved with CPLEX. It has a Visual Basic front end, developed internally by the Berkeley Lab DER-CAM team, permitting ease of data and parameter entry.

\subsection{DER-CAM Applications}

Studies done using DER-CAM include:

- The modeling of a hypothetical microgrid in San Diego based on a collection of businesses in that city (Bailey, 2002).

- An integration of DER-CAM with Geographical Information Systems (GIS) (Edwards, 2002) to examine DER potential in a San Diego neighborhood.

- The effects of a carbon tax on the adoption of DER technologies (Marnay, 2002).

- A DER case studies project that included DER-CAM model verification (Bailey, 2003).

\subsection{The Need for More Detailed Modeling}

While DER-CAM is an excellent tool for determining candidate DER systems and dispatch schedules, there are some details of the process that require closer examination.

\subsubsection{DER Technology Modeling}

The technology descriptions in DER-CAM are simple by necessity, as DER-CAM is faced with the computationally intensive task of determining the optimal performance of

${ }^{11}$ GAMS is a proprietary software product used for high-level modeling of mathematical programming problems owned by the GAMS Development Corporation (http://www.gams.com) 
any possible DER system in order to determine the optimal DER solution. Before a DER system is selected in the field, it would be necessary to consider a more detailed model of the proposed DER system to confirm that its performance is similar to that predicted by DER-CAM. SESU is well suited to this task.

\subsubsection{DER Dispatch Schedules}

The dispatch schedules determined by DER-CAM are based on DER-CAM's a priori knowledge of the site's energy loads for the entire year and the assumption that DER equipment is $100 \%$ reliable. In reality, historic load data can be used to predict loads, although actual loads will vary, and optimal dispatch cannot be determined in advance of actual events throughout the year. 



\section{Designing an EM and a Microgrid}

The complimentary capabilities of DER-CAM and SESU are well suited for the task of designing an EM for a given microgrid and for determining candidate microgrid configurations. Together they can be used to develop a DER system, including EM, for a potential microgrid site such at the CERTS Demonstration Microgrid.

\subsection{SESU as a Tool for EM Design}

SESU provides a platform for testing candidate EMs on a model of a proposed or existing microgrid. The following procedure can be used to compare the costs and benefits of various candidates:

1) For each candidate EM, determine the feasibility and costs of implementation and ongoing reconfiguration. This will include the cost of sensors, access to information via the internet, communication infrastructure, data storage, and information processing.

2) Implement each candidate EM on the proposed microgrid in SESU. Assess the performance of the microgrid over the desired time interval (typically one or more years). Performance may be measured by such a metric as expected energy costs or some more detailed statistical description of energy costs.

3) Add the costs of EM implementation to the energy costs to determine the overall energy costs for the microgrid.

4) Choose the most attractive EM. Perhaps do more SESU runs to refine the performance of this EM and other EMs that resulted in comparable overall energy costs.

5) Test the robustness of the selected EM to unanticipated conditions.

\subsection{DER-CAM as a Tool for Microgrid Configuration Selection}

DER-CAM has already been used as a tool for selecting optimal DER system configuration given a site's load, tariff, and equipment option data (Section 8.2). Sensitivities can be done to key factors in DER-CAM to determine a set of candidate optimal configurations.

This set of candidate configurations can then be modeled in SESU and assessed in conjunction with actual EMs to determine which combined configuration/controller system performs the best. The results of this procedure can then be used to build the actual microgrid and EM. 



\section{Example EM Tests From Prior Case Study Work}

SESU can be used to assess various aspects of microgrid design and/or operations that are not amenable to analysis by DER-CAM or other general planning tools. One such consideration is the consequences of generator unreliability, which are highly complex when demand charges are involved. An example inspired by prior research at the Berkeley Lab here illustrates the way SESU can help answer difficult questions and also shows some of the concepts and procedures discussed in preceding chapters.

One of the case studies reported in (Bailey, 2003) and examined in more detail in (Firestone, 2003) was the BD Biosciences Pharmingen life-sciences supply manufacturer in San Diego, California. The site currently has two $150 \mathrm{~kW}$ natural gas engines with heat recovery on-site to offset electrical and heating loads.

Neither the developer for this project nor the DER-CAM team predicted the affect of generator reliability on demand charges. Both assumed, for the purpose of cost assessment, that the generators were $100 \%$ available, and that deviations from this would not affect costs considerably. For example, if the generators were only available $95 \%$ of the time, then electricity purchase from the utility would increase by approximately $5 \%$, as would utility electricity bills. This would be balanced by a $5 \%$ decrease in fuel and operation and maintenance costs on the generators during the time when they were offline. These assumptions miss the significant affect of demand charges on utility bills. For example, if both generators were to go out simultaneously during the on-peak period, even for a short time, then a large demand charge would be incurred. Note that as electricity purchases decline following installation of on-site generation, the fraction of the bill determined by fixed charges and demand charges will likely increase. This effect can be extra powerful if equipment failures result in demand charges akin to those incurred prior to the displacement of significant energy purchases.

DER-CAM was used to model this system. For the month of June, DER-CAM predicted a peak utility demand of $159 \mathrm{~kW}$ based on the $100 \%$ reliability assumption. This value is simply the maximum of the hourly average weekday electricity loads (from historic data) less the $300 \mathrm{~kW}$ capacity of the natural gas engines. Because this peak demand occurs during San Diego Gas and Electric's (SDG\&E) on-peak hours, total monthly demand charges at rates current at the time of this report are $\$ 17.72 / \mathrm{kW}^{12}$, and the predicted demand charges for June of the test year would be $\$ 2,800$.

While this serves as an appropriate first estimate of costs, it neglects several system details. One is the imperfect reliability of generators discussed above. Another is the deviation of daily load profiles from the monthly average daily load. Demand charges will be incurred from the maximum load during the month, not the maximum of averaged loads. These deviations from an average loads, as well as the probabilistic behavior of

\footnotetext{
${ }^{12}$ Current SDG\&E on-peak summer rates are $\$ 0.076 / \mathrm{kWh}, \$ 10.85$ on-peak demand, $\$ 6.87$ non-coincident demand (the site's non-coincident demand is during the on peak hours). The on-peak hours are 11 AM to 6 PM. Tariff schedules are available at http://www.sdge.com/
} 
generators, invite a stochastic examination of the system in order to derive a better estimate of monthly demand charges. This more detailed estimate of monthly demand charges can be accomplished by SESU. Figure 8 shows the SESU workspace for this example, and the following subsections describe the workspace modules.

\subsection{Device Modules}

As only the demand charges were being considered in this execution of SESU, only the electrical microsources needed to be represented. Two instances of the $150 \mathrm{~kW}$ natural gas engine module were generated in SESU and placed inside of a generator bank module for graphical simplicity. Figure 6 shows the inside of the generator bank module including the two natural gas engine modules. The input to each engine module is the setpoint command from the EM. The output from each module is the current electrical output, the status of the generator (on or off-due-to-fault), and the rated capacity of the generator. Thus the generator bank accepts a setpoint signal and outputs the total electrical output from the two generators, the status of each generator, and the rated capacity of each generator.

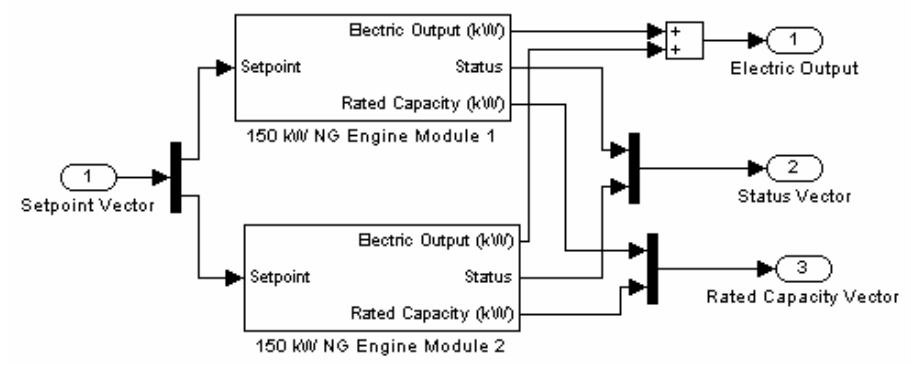

Figure 6: Inside of Generator Bank Including Natural Gas Engine Modules

Table 1 lists the parameter values used for both instances of the module. Note that because this example considers only costs associated with demand charges, it is unnecessary to examine the fuel consumption of the generators, electricity $(\mathrm{kWh})$ purchases, or natural gas purchases over the month. Instead, it is assumed that during onpeak hours, the generators are always running if available and producing as much electricity as needed, up to their rated capacity of $150 \mathrm{~kW}$ each. Thus, to determine the monthly demand charge, only the monthly peak demand needs to be determined.

Table 1: Natural Gas Engine Module Parameters

\begin{tabular}{|l|c|}
\hline Capacity (kW) & 150 \\
\hline Reliability & $\begin{array}{c}\text { 92\%, 96\%, and 100\% for } \\
\text { different trials }\end{array}$ \\
\hline Expected outage length (hours) & 8 \\
\hline $\begin{array}{l}\text { Time for generator to ramp } \mathbf{8 0} \% \text { of } \\
\text { the way from current load to a } \\
\text { new setpoint (minutes) }\end{array}$ & 1 \\
\hline
\end{tabular}


Engine outage is represented as a Markov chain process in the natural gas engine modules. Based on the parameters of availability and expected outage length, the Markov probabilities can be determined ${ }^{13}$. The four probabilities to be determined are:

- $\mathrm{P}_{\text {off-on: }}$ the probability of a generator becoming unavailable at a given time step if it is available at the previous time step.

- $\mathrm{P}_{\text {on-on: }}$ the probability of a generator remaining available at a given time step if it is available at the previous time step.

- $\mathrm{P}_{\text {off-off: }}$ the probability of a generator remaining unavailable at a given time step if it is unavailable at the previous time step.

- $\mathrm{P}_{\text {on-off: }}$ the probability of a generator becoming available at a given time step if it is unavailable at the previous time step.

\subsection{The Load Module}

The load module reports the site electrical load at each time step. Because demand charges are only incurred during on-peak hours, only these hours were considered in this model. Modeling electric loads as a stochastic variable was done by determining the load at each timestep by Eq. (1)

$$
\text { Load }(\text { day, time })=\alpha(\text { day }) \bullet \text { AverageLoad(time })+\beta(\text { day, time })
$$

where

- $\operatorname{Load}($ day, time) is the actual load on a given day at a given time.

- AverageLoad(time) is the monthly average load at a given time, based on historic data.

- $\quad \alpha(d a y)$ is a daily load scalar to represent daily fluctuations in load profile due to fluctuations in weather, production loads, or other phenomenon.

- $\quad \beta$ (day, time) is a disturbance term to represent load dips and peaks throughout each day due to the turning on and off of large machinery or other phenomenon.

Figure 7 shows the average load profile for the on-peak hours of June, based on site data from 2001. Base on the site data provided, $\alpha$ was estimated as a uniform random variable on the range $[0.8,1.2]$ and $\beta$ was estimated as a uniform random variable on the range [20,20].

\footnotetext{
${ }^{13}$ In practice, it is difficult to obtain equipment performance detailed enough to estimate these probabilities or to confirm that the structure of this model of availability is accurate. The expected outage length will vary with the structure of service provisions (mechanics on-site versus an off-site maintenance provider) as well as the type of use of the equipment (base-loading versus load following). The eight hour average outage length used in this study is a rough estimate based on conversations with off-site maintenance providers.
} 


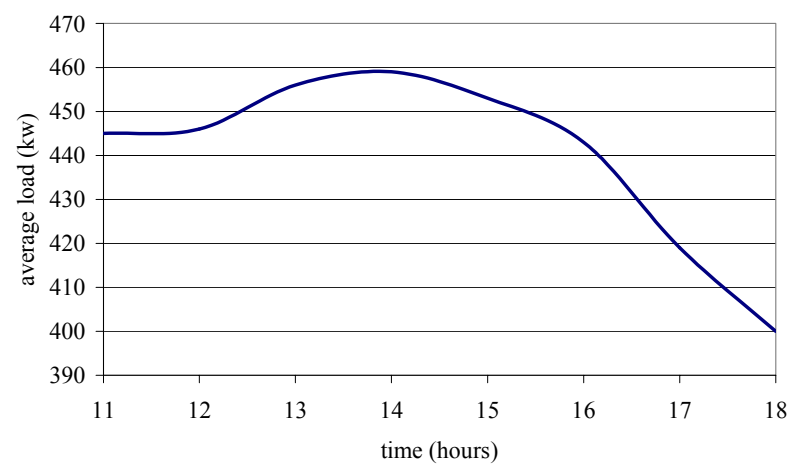

Figure 7: Average Load Profile for June On-peak Hours

\subsection{The EM Module}

The EM module assesses the state of the system at each timestep and dispatches the device modules accordingly. The inputs to the EM are the current site electrical load, and the status and capacity of each generator. Based on this information, the EM determines what setpoint signal to send to each generator. In this example, the generators are always on during on-peak hours, and because the site load is more than the combined capacity of the two generators, the setpoint of each generator is always $150 \mathrm{~kW}$.

\subsection{The Demand Charge Module}

The demand charge module determines the electricity purchased from the utility at each timestep during the month, selects the maximum demand, and multiplies this value by the utility demand charge to output the monthly demand charge for the site.

\subsection{The SESU Workspace}

Figure 8 shows the entire SESU workspace, which contains the generator bank, the load module, the EM module, and the demand charge module. Maximum demand is defined as the largest average load drawn from the utility during a 15-minute interval.

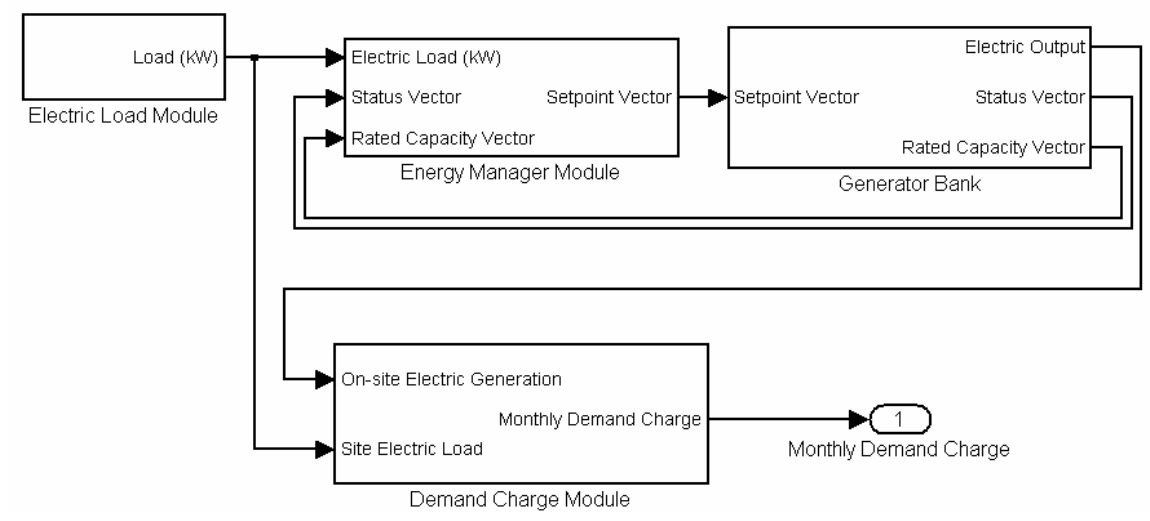

Figure 8: SESU Workspace 


\subsection{Unreliability Results}

Because of the stochastic behavior of the load (determined by $\alpha$ and $\beta$ ) and the generators (determined by the Markov process), a Monte Carlo simulation was employed to determine the probabilistic description of the demand charges for the month of June. For each of the three reliability cases (92\%, 96\%, and 100\%), 5000 SESU runs were executed. In all cases, the expected length of outage was eight hours. Each run examined 22 weekday on-peak intervals, representing the weekdays in the month of June. The demand charge from each run was stored in bins of $\$ 50$ increments. Probability density functions ${ }^{14}$ were generated from the results and are presented in Figure 9 through Figure 11. The expected values for each of these cases is reported in Table 2.

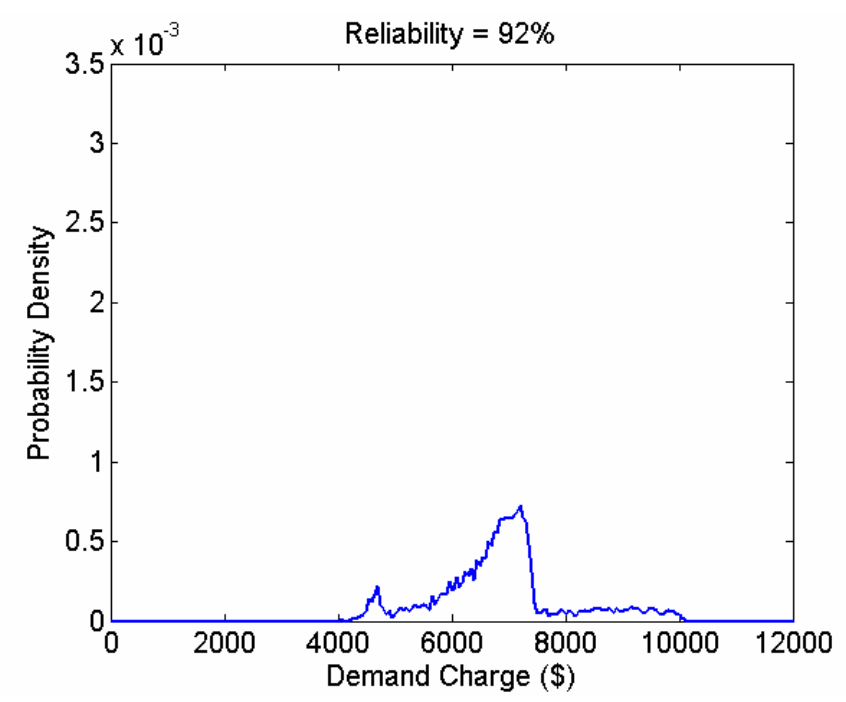

Figure 9: Probability Density for $\mathbf{9 2 \%}$ Reliable Engine

\footnotetext{
${ }^{14}$ For a probability density function, $\mathrm{f}(\mathrm{x})$, the probability of the demand charge being valued between a and $\mathrm{b}$ is

Probability $(\mathrm{a}<$ DemandCharge $<\mathrm{b})=\int_{\mathrm{a}}^{\mathrm{b}} \mathrm{f}(\mathrm{x}) \mathrm{dx}$
} 


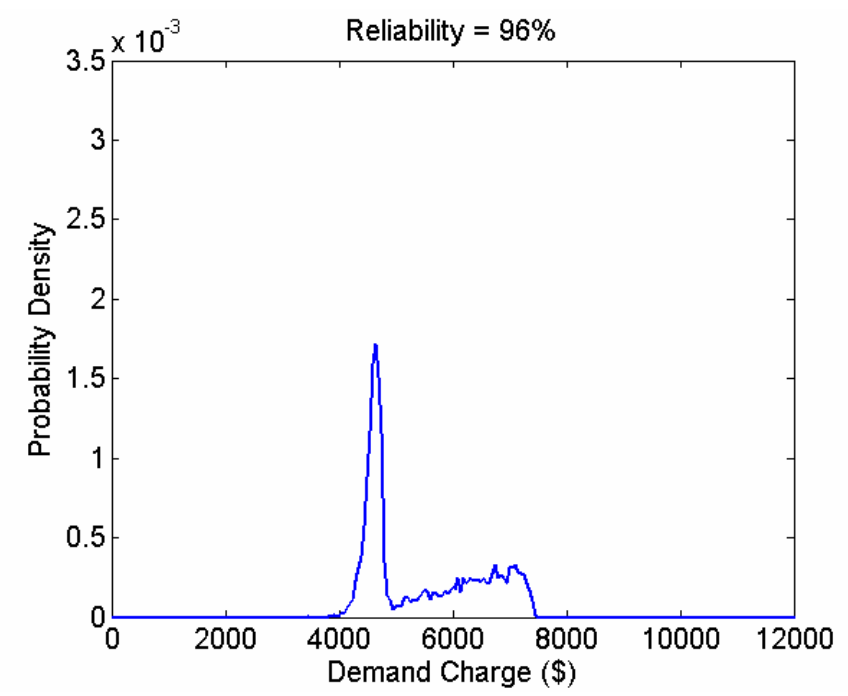

Figure 10: Probability Density for $96 \%$ Reliable Engine

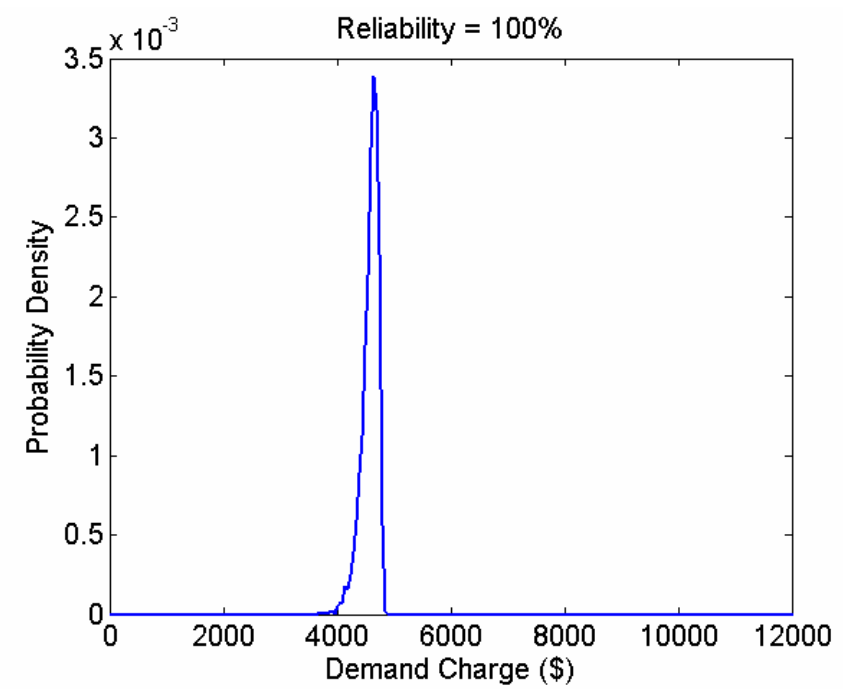

Figure 11: Probability Density for $100 \%$ Reliable Engine

Table 2: Expected Demand Charges

\begin{tabular}{|l|c|}
\hline & $\begin{array}{c}\text { Expected Value of Demand } \\
\text { Charge For June }\end{array}$ \\
\hline DER-CAM and Developers & $\$ 2,880$ \\
\hline SESU: 92\% Reliabilty & $\$ 6,880$ \\
\hline SESU: 96\% Reliabilty & $\$ 5,450$ \\
\hline SESU: 100\% Reliabilty & $\$ 4,580$ \\
\hline
\end{tabular}

SESU has demonstrated that the expected demand charge for the month of June was underestimated by DER-CAM by $\$ 1,700$ to $\$ 4,000$, under the perfect reliability 
assumption used. Even in the case of $100 \%$ reliability, the demand charges are still expected to be $\$ 1,700$ above those predicted by DER-CAM because of stochastic variation in loads ${ }^{15}$. At this site, savings expected by the site were $\$ 70,000$ year. A more comprehensive SESU study could determine how much the site's savings estimates would be decreased due to imperfect engine reliability and stochastic loads. Only if the $\alpha$ term has a value of one and the $\beta$ term has a value of zero in Eq.(1) would the expected demand charge be at the level used in the BD Biosciences analysis.

When reliability is less than $100 \%$, expected demand charges increase, as does the potential for an occasional extremely large demand charge. For the case of $92 \%$ reliability, there is a $40 \%$ chance that the demand charge will be greater than $\$ 7,000$, a $13 \%$ chance that it will be greater than $\$ 8,000$ and a $6 \%$ chance that it will be greater than $\$ 9,000$. Table 3 summarizes these statistics for the $92 \%$ and $96 \%$ reliability cases. This cost uncertainty eliminates one of the benefits of DER: price stability. Using demand control to reduce this uncertainty is examined in the following sections.

Table 3: Demand Charge Variation Statistics

\begin{tabular}{|c|c|c|}
\hline & \multicolumn{2}{|c|}{ Probability } \\
\hline $\begin{array}{c}\text { Demand } \\
\text { Charge } \\
\text { Greater Than }\end{array}$ & $\begin{array}{c}\text { 92\% Engine } \\
\text { Reliability }\end{array}$ & $\begin{array}{c}\mathbf{9 6 \%} \text { Engine } \\
\text { Reliability }\end{array}$ \\
\hline $\mathbf{\$ 6 , 0 0 0}$ & 0.82 & 0.34 \\
\hline $\mathbf{\$ 7 , 0 0 0}$ & 0.41 & 0.10 \\
\hline $\mathbf{\$ 8 , 0 0 0}$ & 0.13 & 0.00 \\
\hline $\mathbf{\$ 9 , 0 0 0}$ & 0.07 & 0.00 \\
\hline $\mathbf{\$ 1 0 , 0 0 0}$ & 0.00 & 0.00 \\
\hline
\end{tabular}

\subsection{An EM Test With Load Control}

For the $92 \%$ reliability case above, the probability of demand charges greater than $\$ 8,000$ for the month of June is significant. These large charges are incurred when both engines are simultaneously off-line. If load control could be used to lower site electricity demand during these times, then a significant measure of price risk could be eliminated. SESU can be used to analyze the benefits of demand control in this situation.

For this example, it is assumed that the site is capable of curtailing $100 \mathrm{~kW}$ of electric load. Although no cost is associated with this curtailment, the site is only willing to curtail at most once per week, and only up to 3 hours. It is assumed that no advance warning is required for this curtailment and that the full curtailment takes place instantaneously.

\footnotetext{
15 This large discrepancy is approximately explained as follows:

For a $100 \%$ reliable DER system, the site expects approximately $160 \mathrm{~kW}$ maximum electricity demand (460 kW maximum consumption $-300 \mathrm{~kW}$ on-site generation). For demand charges of $\$ 17.72 / \mathrm{kW}$, this is a monthly demand charge of $\$ 2800$.

However, when loads are modeled stochastically, the maximum consumption of electricity is closer to $120 \%$ of the maximum average load. This is a maximum demand of $552 \mathrm{~kW}$, and a maximum utility purchase of $252 \mathrm{~kW}$. For demand charges of $\$ 17.72 / \mathrm{kW}$, this is a monthly demand charge of $\$ 4500$.
} 
Figure 12 shows the SESU workspace with demand control. Here, the load module has been replaced with a critical load module and a curtailable load module.

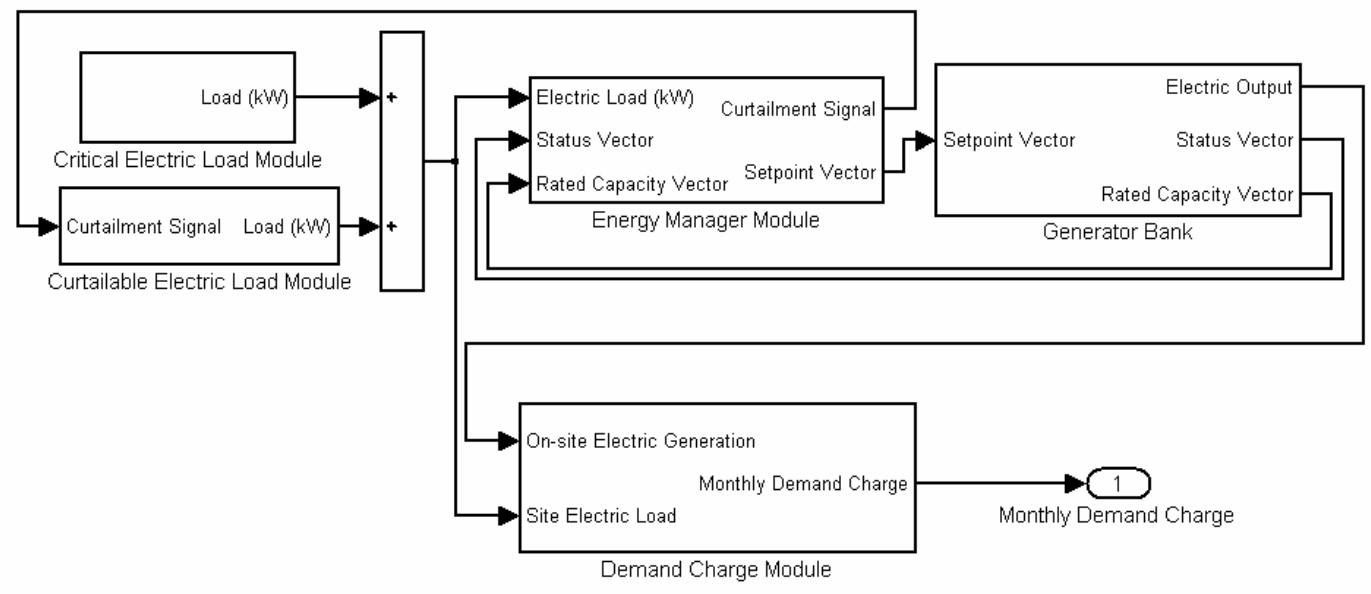

Figure 12: SESU Workspace for microgrid with Load Control

The critical load module requests loads based on Eq. (2)

$$
\text { Load }(\text { day, time })=\alpha(\text { day }) \bullet \text { AverageLoad }(\text { time })+\beta(\text { day, time })-100
$$

where Load, AverageLoad, $\alpha$ and $\beta$ are defined as above. The curtailable load module requests a load of $100 \mathrm{~kW}$ if no curtail signal is given from the EM and $0 \mathrm{~kW}$ if the curtail signal is given.

\subsection{Results with Load Control}

The energy manger module was reprogrammed to request curtailment up to once per week, for up to three hours if both generators are off-line. The probability densities are given in Figure 13, and the expected values and variation statistics are given in Table 4.
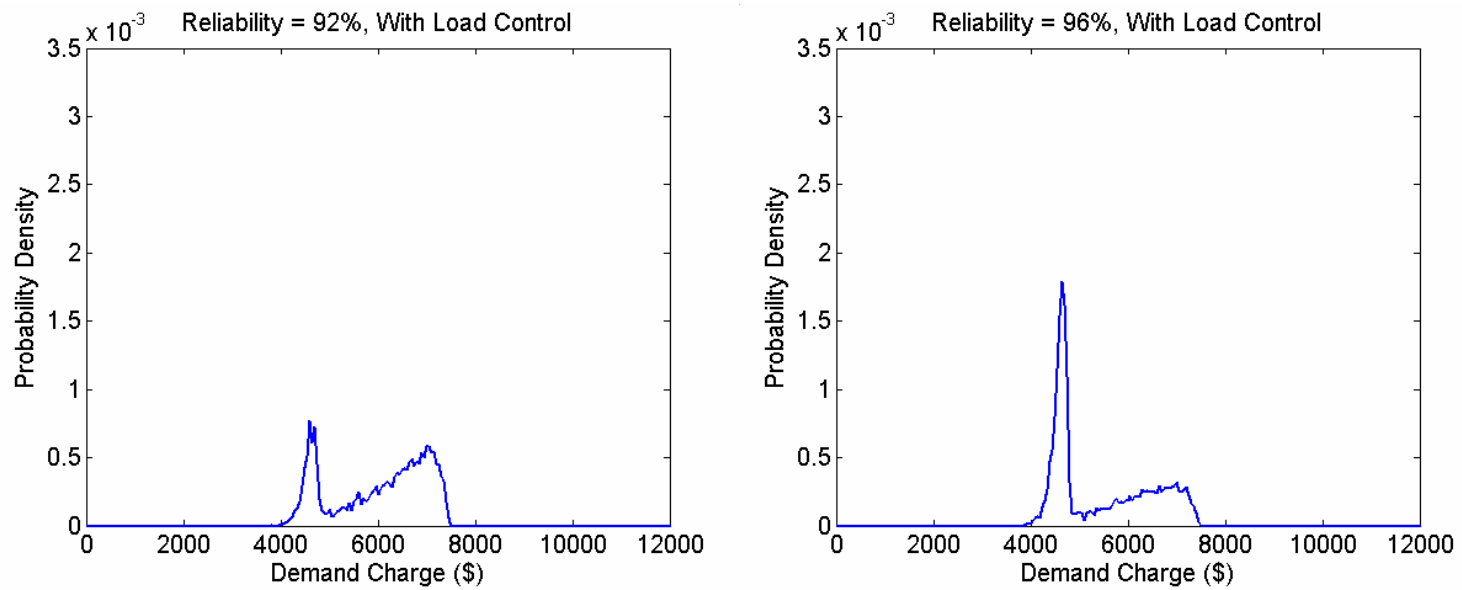

Figure 13: Probability Densities for Engine Reliability of 92\% (left) and 96\% (right) with Load Control 
Table 4: Demand Charge Variation Statistics and Expected Value with Load Control

\begin{tabular}{|c|c|c|c|c|}
\hline \multirow{3}{*}{$\begin{array}{c}\text { Demand } \\
\text { Charge } \\
\text { Greater Than }\end{array}$} & \multicolumn{4}{|c|}{$\begin{array}{l}\text { Probability } \\
\end{array}$} \\
\hline & \multicolumn{2}{|c|}{ 92\% Engine Reliability } & \multicolumn{2}{|c|}{ 96\% Engine Reliability } \\
\hline & $\begin{array}{c}\text { Without Load } \\
\text { Control }\end{array}$ & $\begin{array}{c}\text { With Load } \\
\text { Control }\end{array}$ & $\begin{array}{c}\text { Without Load } \\
\text { Control }\end{array}$ & $\begin{array}{c}\text { With Load } \\
\text { Control }\end{array}$ \\
\hline$\$ 6,000$ & 0.82 & 0.58 & 0.34 & 0.34 \\
\hline$\$ 7,000$ & 0.41 & 0.18 & 0.10 & 0.10 \\
\hline$\$ 8,000$ & 0.13 & 0.00 & 0.00 & 0.00 \\
\hline$\$ 9,000$ & 0.07 & 0.00 & 0.00 & 0.00 \\
\hline$\$ 10,000$ & 0.00 & 0.00 & 0.00 & 0.00 \\
\hline
\end{tabular}

For engines with $96 \%$ reliability, the occurrence of simultaneous engine faults is rare enough that the load control implemented here offers no noticeable benefit. However, for engines with $92 \%$ reliability, these results demonstrate the ability of the EM to affect a ceiling on the demand charges and to reduce the expected demand charges by avoiding large demand when both generators are off-line. The value of these improved results must we weighed against the cost of implementing demand control to determine if this control strategy is desirable. In this example, SESU has been used to assess the benefits of a simple demand response rule. Of course, much more sophisticated rules could have been considered, and while it may seem pedestrian in some aspects, testing control algorithms in this way does allow reasonable choices to be made between alternatives that are difficult to tackle analytically. The approach also allows for ready comparisons of performance between alternatives, and with idealized solutions, such as DER-CAM results. Most importantly, tradeoffs between costs and benefits of complexity can be gauged. The examples shown here involving demand charges were not randomly chosen, but were rather suggested by prior analysis of installed DG systems and hypothetical examples. Experience has shown that demand charges are powerful drivers of outcomes. Many other control choices will undoubtedly offer much less dramatic results and selecting a robust option will be consequently more difficult. 



\section{Dispatch for a Two Degree of Freedom ${ }^{17}$ Microgrid}

Compared to the issues discussed in Chapter 10, real-time microgrid optimization is straightforward. If a microgrid is to be optimized to minimize energy costs, sunk costs are ignored and operating costs must be minimized at every point in time. At each timestep, the EM must make dispatch decisions for the coming time-step, and possibly make planning decisions for future time-steps. As discussed in Section 3.1, the cost of energy may not be deterministic in real-time. In such cases, the EM must make cost-minimizing decisions without knowing the actual cost of energy until later.

The most common example of this is demand charges. Demand charges are incurred for the maximum power consumption in a given time range. If a site anticipates periods of relatively high consumption at the end of the month, then it will not consider monthly demand charges in its decision-making during the period of relatively low consumption at the beginning of the month and vice versa. However, if a site does not anticipate any high consumption periods during the month, then demand charges must be considered at all times.

An "optimal" control strategy for distributed electrical generation systems has been proposed (Curtiss, 2003). Here, to determine the dispatch for the coming time-step, the EM predicts the electricity demand and considers all possible dispatch decisions and the energy costs associated with each. The EM always assumes that the current time step is the last of consideration for the demand charge and can thus assess costs deterministically. The dispatch decision that incurs the minimum costs is selected. This is a real-time optimization strategy as discussed in Section $4.1^{18}$.

For time-steps not at the end of the month, this strategy inherently assumes that electricity demand for the rest of the month will not be greater than the current maximum demand. This prediction is not always warranted. The rest of this section provides examples to illustrate how decision-making is affected by predictions when demand charges are present.

\subsection{A Simple Microgrid}

Consider a microgrid that consists of one $60 \mathrm{~kW}$ microturbine and one $60 \mathrm{~kW}$ natural gas engine, both with heat recovery. The microturbine is part of an integrated CHP unit with a system efficiency ${ }^{19}$ of $73 \%$ at rated capacity while the natural gas engine waste heat is

\footnotetext{
${ }^{17}$ A degree of freedom is an independent dispatch decision to be made at each timestep. Dispatch decisions to individual generators are all degrees of freedom. Additional degrees of freedom come from storage and end-use decisions.

${ }^{18}$ For more complex scenarios (such as more dispatch decisions), optimization algorithms could beneficially replace the enumeration method used here.

${ }^{19}$ Here, system efficiency is defined as

SystemEfficiency $=\frac{\text { ElectricalEnergy }(\mathrm{kW})+\text { RecoveredHeat }(\mathrm{kW})}{\text { FuelConsumed }(\mathrm{kW})}$
} 
only recovered at the exhaust, and not at the radiator. The natural gas engine has a system efficiency of 54\% at rated capacity. Both of these engines can be operated in the range of $50 \%$ to $100 \%$ of their rated capacity. The microgrid is connected to the utility grid as well, and pays a time of use (TOU) rate that includes monthly non-coincident demand charges during the on-peak hours. The microgrid has a heat load and can burn natural gas to supplement the recovered heat of the engines. Figure 14 shows the schematic setup of this microgrid.

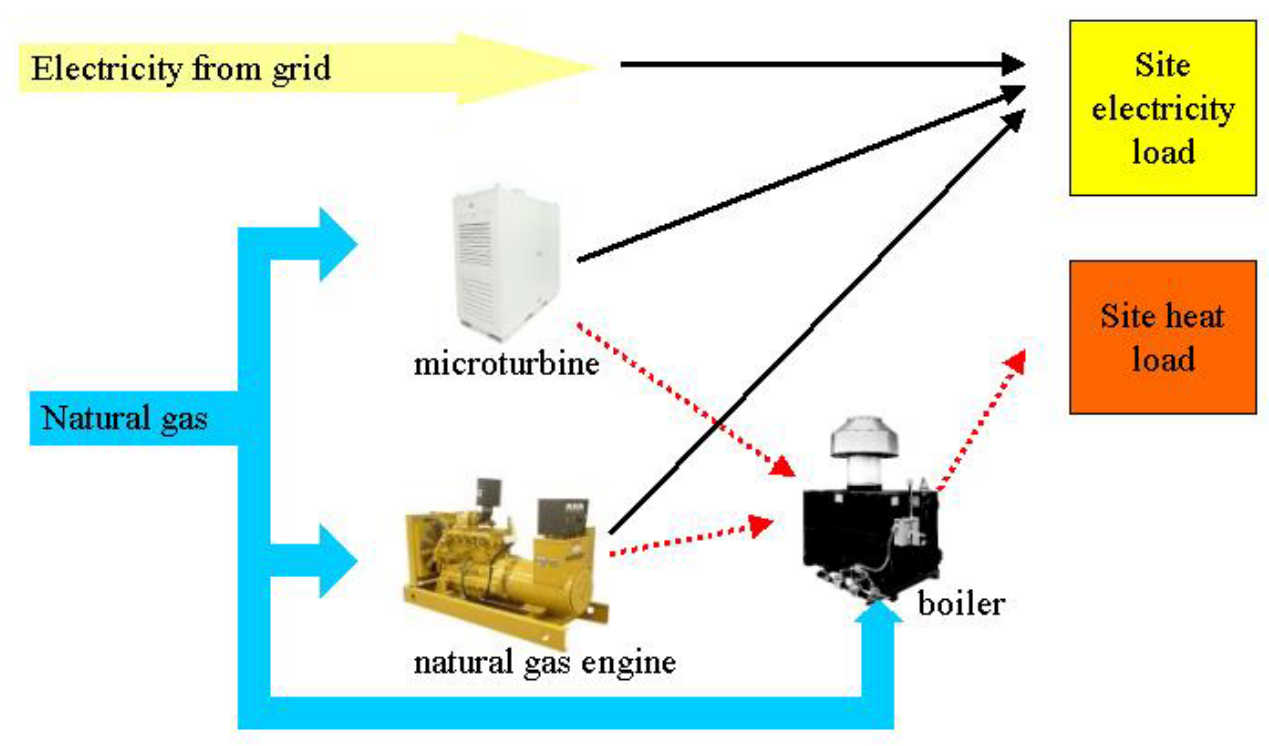

Figure14: Microgrid Schematic

Engine performance data for the Capstone C60 Integrated CHP $60 \mathrm{~kW}$ microturbine and the Katolight SP series $60 \mathrm{~kW}$ natural gas engine were used. A summary of performance data used for modeling in this example is listed in Table 5. Utility and fuel prices are representative of the San Diego, California area during the summer of 2003 (Table 6).

Table 5: Microgrid Specifications

\begin{tabular}{l|r|r|}
\hline & $\mathbf{6 0 ~ k W ~ M i c r o t u b i n e ~}$ & $\mathbf{6 0 ~ k W}$ natural gas engine \\
\hline Rated capacity (kWe) & $60^{1}$ & $60^{2}$ \\
\hline Efficiency at $\mathbf{5 0 \%}$ of rated capacity (HHV) & $0.22^{3}$ & $0.26^{2}$ \\
\hline Efficiency at $\mathbf{7 5 \%}$ of rated capacity (HHV) & $0.24^{3}$ & $0.27^{2}$ \\
\hline Efficiency at 100\% of rated capacity (HHV) & $0.25^{1}$ & $0.3^{2}$ \\
\hline Recoverable heat to electrical output ratio & $1.9^{1}$ & $0.8^{3}$ \\
\hline Operation and maintenance costs (\$/kWh) & $0.018^{3}$ & $0.018^{3}$ \\
\hline
\end{tabular}

where FuelConsumed is the higher heating value of natural gas. 
Table 6: Energy Prices

\begin{tabular}{|l|cl|}
\hline On Peak Electricity Prices (\$/kWh) & 0.09 & \\
\hline On Peak Demand Charges (\$/kW) & 17.72 & \\
\hline Natural Gas Prices (\$/GJ) & 5.46 & $($ \$0.576/therm) \\
\hline
\end{tabular}

For any possible combination of operation setpoints for the two generation devices, microgrid operating costs can be determined for a given time-step as the sum of the following:

- electricity purchase from the utility,

- electricity demand charges from the utility,

- natural gas purchase for heating,

- natural gas purchase to fuel the microturbine,

- natural gas purchase to fuel the natural gas engine,

- operation and maintenance costs for the microturbine, and

- operation and maintenance costs for the natural gas engine.

In this example, one one-hour time-step is considered and costs are determined assuming that loads and generator setpoints will be constant for each time-step. For each scenario, the operating costs over possible dispatches are calculated.

From the discussion of Curtiss (2003) above, it is seen that prediction of monthly maximum demand is necessary to predict current operating costs. The EM can make this prediction based on historical data and knowledge of the present system. Once a monthly peak demand is predicted, demand charges at a given time-step are determined from the incremental demand above the maximum demand prediction.

\subsection{Optimization Strategy and Visualization}

The control scheme in this example is a real-time optimization at each timestep. This optimization entails the following steps:

1. Determine the set of possible dispatch states.

2. Assess the system energy costs for each possible dispatch state.

3. Select the state associated with the minimum energy costs and dispatch the system accordingly.

This procedure is illustrated here in contour plots. For this example, there are two parameters in the dispatch state: the dispatch level of the microturbine and the dispatch level of the natural gas engine. The two dimensions of the contour plot correspond to these two decision variables. Colored areas in the contour plot represent the possible dispatch states (each device can be off or running from $50 \%$ to $100 \%$ of its rated capacity). Colors correspond to system energy costs, with darker colors showing lower costs. The optimal dispatch decision is determined from the lowest cost (darkest color) state on the contour plot. 


\subsection{Scenario 1: No Heat Load and No Demand Charge Considerations}

Scenario 1 considers a current site electrical demand of $100 \mathrm{~kW}$, no current site heating demand, and an expected maximum demand for the month of $100 \mathrm{~kW}$. The expected maximum demand implies that demand charges need not be considered in this scenario, because utility electricity purchase cannot be greater than the expected demand. Figure 15 displays the contour plot for Scenario 1. Here, the optimal dispatch is to run the natural gas engine at rated capacity and to not run the microturbine.
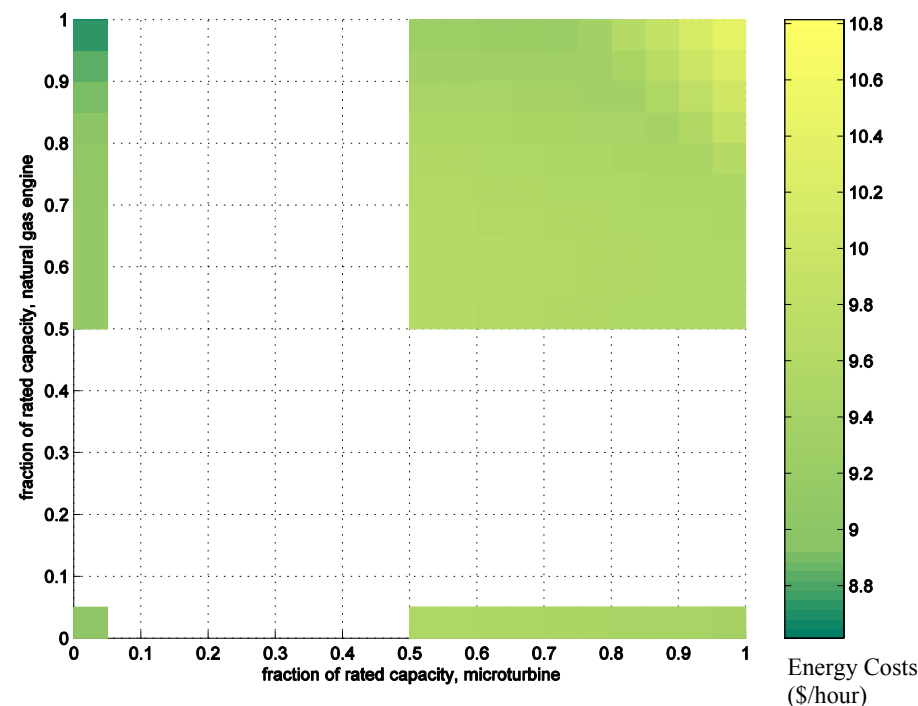

Figure 15: Scenario 1 System Energy Cost Contour (\$/hour)

\subsection{Scenario 2: Heat Load and No Demand Charge Considerations}

Scenario 2 varies from Scenario 1 in that a heat load of $200 \mathrm{~kW}$ is now included. In this scenario, operating the microturbine becomes cost effective because the waste heat offsets the site heating load. Figure 16 displays the contour plot for Scenario 2. Here, the optimal dispatch is to run the natural gas engine at $40 \mathrm{~kW}$ and to run the microturbine at $60 \mathrm{~kW}$. 


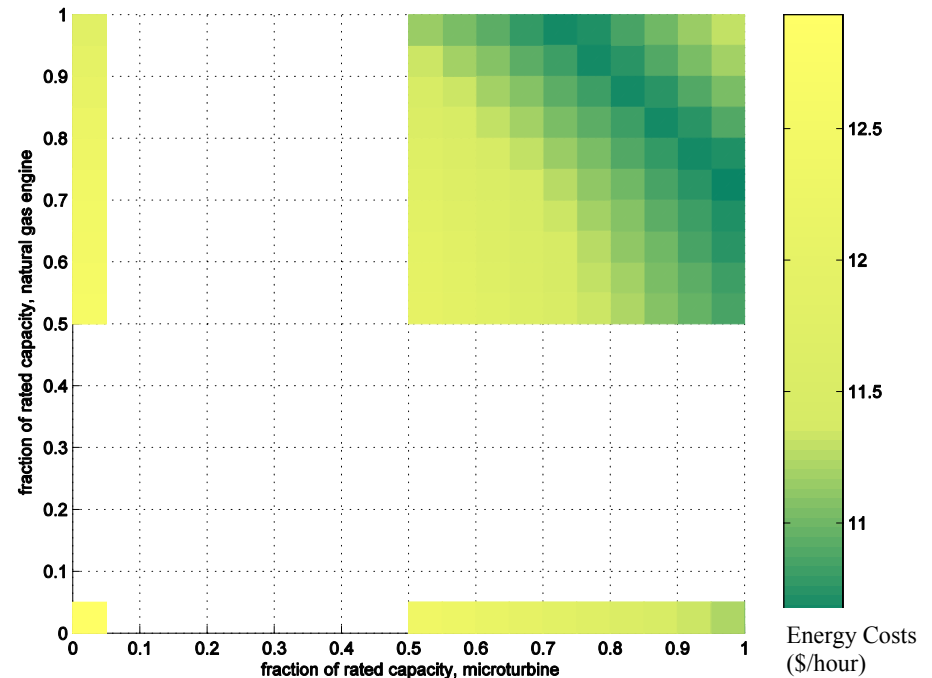

Figure 16: Scenario 2 System Energy Cost Contour (\$/hour)

\subsection{Scenario 3: Demand Charge Considerations but No Heat Load}

Scenario 3 consists of a site electrical load of $1000 \mathrm{~kW}$ and no expected utility purchase for the month, i.e. the site expects to meet all of its electrical demand for the month with on-site generation. Figure 17 displays the contour plot for Scenario 3. Note the extremely large operating costs for dispatching the system to generate less than $100 \mathrm{~kW}$. Here, the optimal dispatch is to run the natural gas engine at $40 \mathrm{~kW}$ and to run the microturbine at $60 \mathrm{~kW}$.

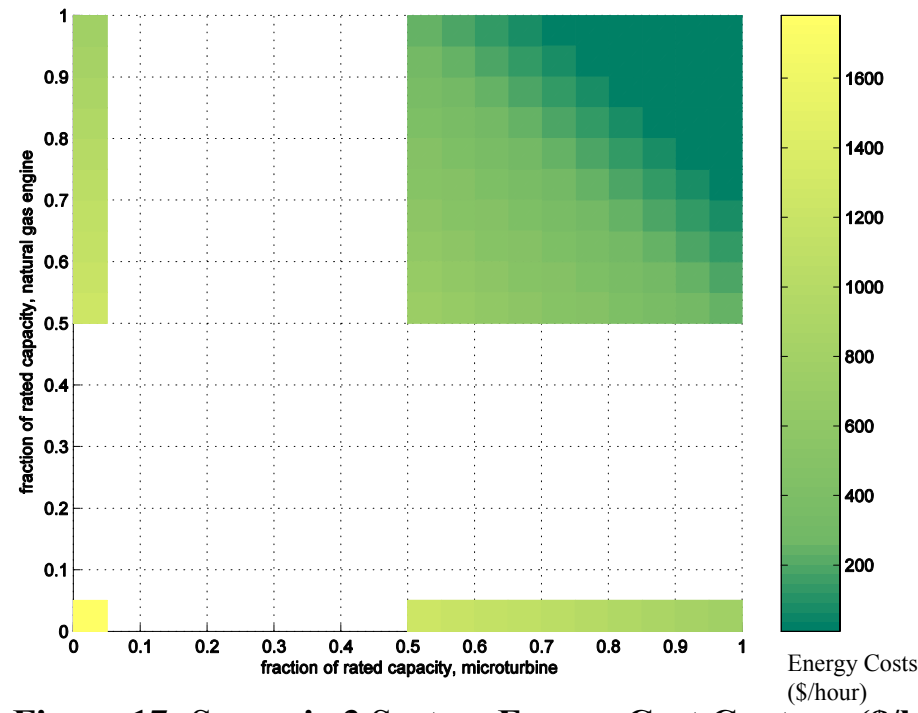

Figure 17: Scenario 3 System Energy Cost Contour (\$/hour) 


\subsection{Scenario 4: Demand Charge Considerations and Heat Load}

Scenario 4 varies from Scenario 3 in that a $200 \mathrm{~kW}$ heat load is now included. Figure 18 displays the contour plot for Scenario 4. Here, the optimal dispatch is to run the natural gas engine at $40 \mathrm{~kW}$ and to run the microturbine at $60 \mathrm{~kW}$.

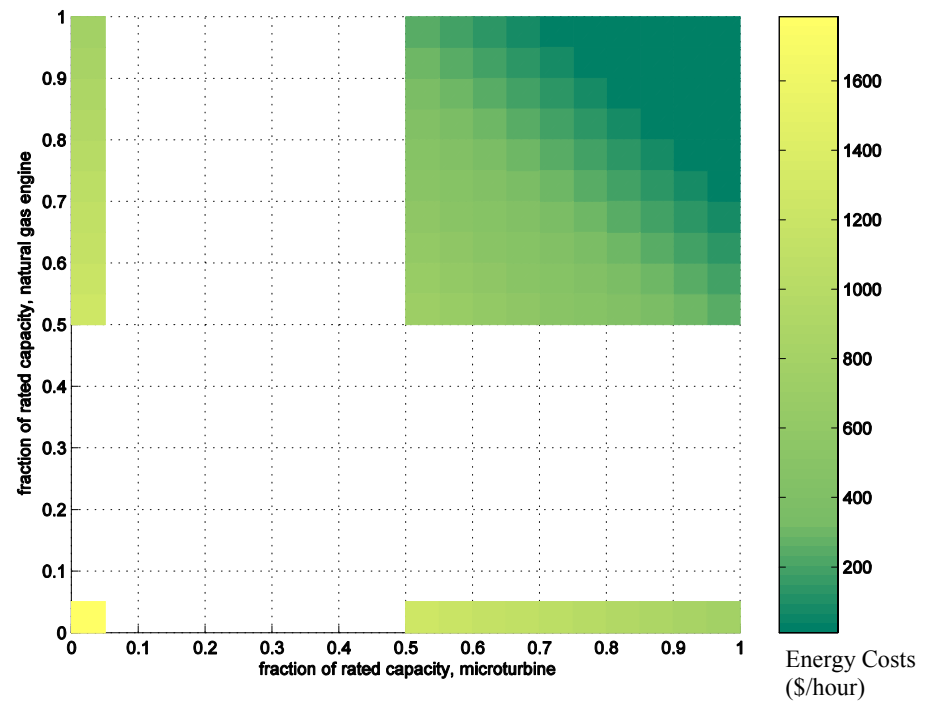

Figure 18: Scenario 4 System Energy Cost Contour (\$/hour)

\subsection{Optimal Control of Complex Microgrids}

This example serves to introduce microgrid optimal control. Even in this simple example, integer constraints (machine can be off, or on in a range that does not start at $0 \%$ ), the non-linearity of efficiency, and demand charges only for demand above previous maximum demand make objective function optimization more difficult than linear programming (see Section 4.1).

For an actual microgrid, there would be many more dispatch degrees of freedom (DOF) than the two in this example (microturbine setpoint and natural gas engine setpoint). Each microsource will contribute at least one DOF. Microturbines with a recuperator that can be used (greater electrical efficiency) or not (greater thermal output) would contribute two DOF. Additional DOF will be contributed by other active DER devices such as chillers, boilers, and storage units if their setpoints can be altered by the controller. If the controller is able to curtail or reschedule certain loads, then each load under consideration would add yet another DOF. More than two control DOF makes contours of operating costs difficult to visualize, yet the optimal dispatch is found in the same manner. From this, the minimum value can be determined and the setpoints at the minimum value can be taken as the optimal dispatch.

Additionally, this example demonstrates the potential for difficulty in determining operating costs in real-time in light of factors such as demand charges which will depend on past, present and future conditions. 


\section{Future Work}

While this report outlines a pragmatic methodology for developing EMs, much of the actual data collection, analysis, and engineering remains to be done. This work could be done both specifically for particular sites such as at candidate CERTS Microgrid demonstration sites and more generally towards the aim of "off the shelf" EMs that are efficient, robust, and expandable. Several areas of immediate interest are:

\subsection{Costs of Acquiring, Transmitting, and Processing Data}

While Chapter 3 discusses the information that affects a microgrid, cost estimates of acquiring, transmitting, and processing data have not been performed. Acquisition costs would include sensors for DER equipment and site conditions, subscriptions to internet data services (if applicable) or the creation of such services. Transmittal costs would be the cost of transmitting data and dispatch to and from the energy manger. The communications infrastructure could take several forms, including a hard-wired ad-hoc network, a wireless network, a self-configuring wireless network (Culler, 2001), or an Internet-based communication network (Rubinstein, 2000). Data processing could be performed on any number of microprocessors or personal computers.

\subsection{Modeling of DER Devices for Use in SESU}

Further work is required to transform SESU into a useful, user-friendly software package. The first step is modeling DER devices. Much data is available on the performance of DER devices, starting with manufacturer specifications and including field-testing. The works of Labinov et.al. (Labinov, 2002), Lipman et. al. (Lipman, 2002), and the American Society of Heating. Refrigeration, and Air Conditioning Engineers HVAC System and Equipment handbook are examples of model sources. This work and original research needs to be transformed into Simulink modules suitable for SESU.

\subsection{Determination of Non-Critical Load Demand Control Costs}

One of the parameters discussed in Section 3.5 was the cost that a site incurs for curtailing or rescheduling a load. It is an arduous, yet potentially beneficial task to determine these costs. Of course, these will be site and load specific, ranging from certain loads for which a site might curtail or reschedule at no perceived cost (such as in the example of Chapter 10) to certain loads that might cost the site significantly to curtail or reschedule. When costs become extremely significant, these loads become critical loads.

As starting point for such studies, Eto et. al. examine the cost of electric reliability in the United States and provides references to numerous studies on this topic (Eto, 2001). These studies do not, however, separate loads by their critical/non-critical status, nor does it examine the cost of electric outages if warning is provided.

Price-responsive load (PRL) programs by utilities offer a testing ground for demand control costs. These programs offer incentives to sites that can curtail some of their loads 
in response to utility signals. Case studies of participants of such programs would be useful in estimating sites' perceived costs of curtailment by examining the incentive level at which they are willing to curtail. Goldman et. al. provide an introduction to this topic (Goldman, 2002).

\subsection{Control Strategies for Complex Systems}

Control strategies are introduced in Chapter 4. More research is needed on effective control and optimization strategies for complex systems. 


\section{Conclusions}

High performance microgrids will require an EM capable of making beneficial decisions based on available information while respecting constraints. The design of EMs will require an understanding of the microgrid system, specification of microgrid objectives and constraints, and assessment of the costs and usefulness of potentially available information. Microgrids can benefit from EMs that integrate the control of energy supply and demand and that predict future conditions. This report introduces some considerations in EM design, a software microgrid modeling platform useful for EM assessment, and a method for developing appropriate EMs. It serves as a framework for the development of the demonstration CERTS Microgrid EM. 



\section{References}

American Society of Heating, Refrigerating and Air-Conditioning Engineers. 2000 "ASHRAE HVAC Systems \& Equipment Handbook 2000 (SI)" ISBN: 1-88341381-8.

Bailey, O., C. Creighton, R. Firestone, C. Marnay, and M. Stadler. 2003 "Distributed Energy Resources in Practice: A Case Study Analysis and Validation of LBNL's Customer Adoption Model” Berkeley, CA: Lawrence Berkeley National Laboratory Report LBNL-52753. (In press)

Culler, D., J. Hill, P. Buonadonna, R. Szewczyk, and A. Woo. 2001. "A NetworkCentric Approach to Embedded Software for Tiny Devices" Berkeley, CA: Intel Research Berkeley Report IRB-TR-01-001.

Curtiss, P. and J. Kreider. 2003. "Recent Developments in the Control of Distributed Electrical Generation Systems" Transactions of the ASME, vol. 125, p. 352-358.

Edwards, J., C. Marnay, E. Bartholomew, B. Ouaglal, A. Siddiqui, and K. HamachiLaCommare. 2002. "Assessment of $\mu$ Grid Distributed Energy Resource Potential Using DER-CAM and GIS" Berkeley, CA: Lawrence Berekeley National Laboratory Report LBNL-50132.

Eto, J., J. Koomey, B. Lehman, N. Martin, E. Mills, C. Webber, and E. Worrell. 2001. "Scoping Study on Trends in the Economic Value of Electricity Reliability to the U.S. Economy" Berkeley, CA: Lawrence Berkeley National Laboratory Report LBNL-47911.

Firestone, R., O. Bailey, C. Creighton, C. Marnay, and M. Stadler. 2003 "A Business Case for On-Site Generation: The BD Biosciences Pharmingen Project" Berkeley, CA: Lawrence Berkeley National Laboratory Report LBNL-52759.

Feero, W., D. Dawson, J. Stevens. "White Paper on Protection Issues of the Microgrid Concept.” CERTS, 2002.

Gibson, Gerald. 2001. "Intelligent Software Agents for Control and Scheduling of Distributed Generation.” California Energy Commission, Public Interest Energy Research Report \#600-01-010.

Goldman, C., M. Kintner-Meyer, G. Heffner. 2002. "Do ‘Enabling Technologies’ Affect Customer Performance in Price-Responsive Load Programs?” Berkeley, CA: Lawrence Berkeley National Laboratory Report LBNL-50328.

Goldstein, L, B. Headman, D. Knowles, S.I. Freedman, R. Woods, T. Schweizer. 2003. "Gas-Fired Distributed Energy Resource Technology Characterizations" Golden, CO: National Renewable Energy Laboratory Report NREL/TP-620-34783. 
Hillier, F., and G. Lieberman. 2001. "Introduction to Operations Research: Seventh Edition”, Boston, MA: McGraw-Hill.

Kozierok, C. 2003. "The PC Guide” http://www.PCGuide.com

Kueck, J.D., R. H. Staunton, S.D. Labinov, and B. J. Kirby. 2003. "Microgrid Energy Management System" Oak Ridge, TN: Oak Ridge National Laboratory Report ORNL/TM-2002/242.

Labinov, S., A. Zaltash, D. Rizy, P. Fairchild, R. DeVault, E. Vineyard. 2002. "Predictive Algorithms for Microturbine Performance for BCHP Systems" Presented at the 2002 ASHRAE Annual Meeting, Honolulu, HI. June 2002.

Lasseter, R., A. Akhil, C. Marnay, J. Stevens, J. Dagle, R. Guttromson, A. S. Meliopoulos, R. Yinger, and J. Eto. 2002. "White Paper on Integration of Distributed Energy Resources: The CERTS MicroGrid Concept" Berkeley, CA: Lawrence Berkeley National Laboratory Report LBNL-50829.

Lipman, T., J. Edwards, and D. Kammen. 2002. "Economic Implications of Net Metering for Stationary and Motor Vehicle Fuel Cell Systems in California" Berkeley, CA. University of California Energy Institute, Program on Workable Energy Regulation Report PWP-092.

Marnay, C., J. Chard, K. Hamachi, T. Lipman, M. Moezzi, B. Ouaglal, and A. Siddiqui.2001. "Modeling of Customer Adoption of Distributed Energy Resources" Berkeley, CA: Lawrence Berkeley National Laboratory Report LBNL-49582.

Marnay, C., J. Edwards, R. Firestone, S. Ghosh, A. Siddiqui and M. Stadler. 2002 "Effects of a Carbon Tax on Combined Heat and Power Adoption by a Microgrid” Berkeley, CA: Lawrence Berkeley National Laboratory Report LBNL-51771.

McNeill, D, and P. Freiberger. 1993. "Fuzzy Logic”, New York, NY: Simon \& Schuster.

Rubinstein, F., S. Johnson, and P. Pettler. 2000. "IBECS: An Integrated Building Environmental Communications System- It's Not Your Father's Network" Berkeley, CA: Lawrence Berkeley National Laboratory Report LBNL-46009.

Siddiqui, A., C. Marnay, J. Edwards, R. Firestone, S. Ghosh, and M. Stadler. 2004. "Effects of a Carbon Tax on Microgrid Combined Heat and Power Adoption" Quantitative Models for Energy Policy, Planning and Management issue of the Journal of Energy Engineering. In press. 


\section{Appendix A: Smart*DER: An EM Platform}

Under contract from the California Energy Commission's (CEC) Public Interest Energy Research (PIER) Program, Alternative Energy Systems Consulting, Inc. produced the report entitled Review of Intelligent Software Agents For Control and Scheduling of Distributed Generation (Gibson, 2001).

This report details the organization of $\operatorname{Smart}^{*}$ DER a software based information and communication system for DER. Smart*DER is subdivided into various agents, individual software programs with specific tasks.

Most of these agents serve the basic, functional necessities of an EM. These agents are:

Owner Interface Agent:

The owner interface agent (OIA) is a graphical user interface (GUI) between the owner/user of the site and the rest of the Smart*DER agents. The OIA is used to monitor the system, execute manual overrides to Smart*DER dispatch, and modify the microgrid description upon which Smart*DER makes its decisions.

Event Manager:

The Event Manager accomplishes periodic activities of Smart*DER. These include data collection and operating schedule updates.

\section{Data Manager:}

The Data Manager archives and retrieves data pertinent to the DER system. This data comes from various sources, such as system sensors (via the Facility Interface Agent) and the internet (via the Internet Agent). Other agents can request data from the DM.

\section{Facility Interface Agent:}

The Facility Interface Agent (FIA) routinely polls all DER asset sensors and sends this information to the Data Manager for storage.

\section{Internet Agent:}

The Internet Agent retrieves pertinent data from the internet such as weather data and energy prices. The Internet Agent also transmits information over the internet as needed.

\section{Data Analyst:}

The Data Analyst provides all data analysis for Smart*DER. This includes prediction of loads and energy prices, the determination of optimal operating schedules for DER assets, and the preparation of site performance reports.

Smart*DER has the additional capability of aggregating several DER sites operating under independent Smart*DER systems. Each Smart*DER system is itself an agent. Smart*DER agents communicate to determine their collective excess capacity and can choose to bid this capacity into the energy market. This aggregation and bidding is done 
through an additional agent located at one of the Smart*DER sites or at a third party location.

Portfolio Manager:

The Portfolio Manager is used to aggregate several DER sites managed by separate Smart*DER systems. The Portfolio Manager can be a more powerful version of one of the OIAs or can be located at a third party site. Individual Smart*DER systems bid their excess generation capacity to the PM by reporting their excess capacity and the cost that would be incurred for providing this (fuel and operation and maintenance). The Portfolio Manager, in turn, aggregates all of the bids and offers one bid to the energy markets.

\section{Smart*DER as a Microgrid EM}

The Smart*DER system could be used as the software package for a microgrid EM. Smart*DER, however, would need the additional capability of optimizing electrical and heating loads simultaneously and dispatching the range of DER technologies, not just electrical generators. Also, Smart*DER would need to be able to incorporate load control for curtailable and reschedulable loads.

The intelligence of the EM, i.e. where the DER control decisions are made, would be in the data analyst. The other agents of Smart*DER would be used to support the Data Analyst and execute the control decisions. DSM Dispatch might take the form of sending control signals to a building energy management system already in place to curtail or reschedule non-critical loads. 